\title{
Second order tail asymptotics for the sum of dependent, tail-independent regularly varying risks
}

\author{
Dominik Kortschak
}

Received: 1 February 2011 / Revised: 3 May 2011 /

Accepted: 19 July 2011 / Published online: 13 August 2011

(C) Springer Science+Business Media, LLC 2011

\begin{abstract}
In this paper we consider dependent random variables with common regularly varying marginal distribution. Under the assumption that these random variables are tail-independent, it is well known that the tail of the sum behaves like in the independence case. Under some conditions on the marginal distributions and the dependence structure (including Gaussian copula's and certain Archimedean copulas) we provide the second-order asymptotic behavior of the tail of the sum.
\end{abstract}

Keywords Dependent random variables - Second order asymptotic · Regularly varying marginals $\cdot$ Copulas $\cdot$ Tail independence

AMS 2000 Subject Classifications $62 \mathrm{E} 20 \cdot 62 \mathrm{P} 05$

\section{Introduction}

Assume that $X_{1}, \ldots, X_{n}$ are dependent random variables, which have a marginal distribution $F$ that is regularly varying with index $\alpha$. If further these random variables are pairwise asymptotic independent. Then (see e.g. Davis and Resnick 1996)

$$
\lim _{u \rightarrow \infty} \frac{\mathbb{P}\left(X_{1}+\cdots+X_{n}>u\right)}{\mathbb{P}\left(X_{1}>u\right)}=n
$$

The author was supported by the Swiss National Science Foundation Project 200021-124635/1.

D. Kortschak $(\varangle)$

University of Lausanne, Lausanne, Switzerland

e-mail: dominik.kortschak@unil.ch 
For independent random variables it is shown in Omey and Willekens (1986) (see also Albrecher et al. 2010, for a recent survey), that under some regularity conditions on $F$ and for $\alpha>1$, the second order approximation is

$$
\mathbb{P}\left(X_{1}+\cdots+X_{n}>u\right)=n \bar{F}(u)+2\left(\begin{array}{l}
n \\
2
\end{array}\right) \mathbb{E}\left[X_{i}\right] f(u)+o(f(u)), \quad u \rightarrow \infty,
$$

where $f$ is the probability density function of $F$ and $\bar{F}(x):=1-F(x)$. A heuristic argument suggests that the sum is large if one component is large and the others are behaving normally, hence

$$
n \bar{F}\left(u-(n-1) \mathbb{E}\left[X_{1}\right]\right)
$$

is a better approximation than $n \bar{F}(u)$, this argument is verified in Albrecher et al. (2010). In the dependent case, it is natural to assume that replacing the mean in Eq. 2 by a conditional mean leads to a better approximation. A Taylor argument then suggests that the second-order asymptotics is given by

$$
\mathbb{P}\left(X_{1}+\cdots+X_{n}>u\right)=n \bar{F}(u)+(1+o(1)) f(u) \sum_{i=1}^{n} \mathbb{E}\left[\left(S_{n}-X_{i}\right) \mid X_{i}=u\right]
$$

where $S_{n}:=X_{1}+\cdots+X_{n}$. However, for a given dependence structure it is not obvious how to evaluate $\mathbb{E}\left[\left(S_{n}-X_{i}\right) \mid X_{i}=u\right]$ and the determination of the asymptotic behavior can be quite tedious. In this paper we provide conditions under which Eq. 3 is valid.

An interesting application of second order asymptotics is Monte Carlo simulation. Whereas the first order asymptotics are used to study the efficiency of estimators, second order estimates can lead to a better understanding of these estimators. For example for the sum of independent random variables Asmussen and Kroese (2006) define the estimator

$$
Z_{A K}(u):=n \bar{F}\left(\left(u-S_{n-1}\right) \vee M_{n-1}\right)
$$

Heuristically, one can see the connection to second order asymptotic approximation:

$$
n \bar{F}\left(\left(u-S_{n-1}\right) \vee M_{n-1}\right) \approx n \bar{F}\left(\left(u-S_{n-1}\right)\right) \approx n \bar{F}\left(\left(u-(n-1) \mathbb{E}\left[X_{1}\right]\right)\right) .
$$

The rest of the paper is organized as follows. In Section 2 we review basic concepts of dependent random variables and regularly varying distributions further we introduce some key Assumptions which either only depend on the marginal distribution or on the copula and the index of regular variation. In Section 3 we derive the second order asymptotics under technical conditions. In Sections 4-6 we present three families of copulas which fulfill these conditions. Further we provide numerical examples in Section 7. Finally the proofs are provided in the Appendices. 


\section{Preliminaries and notations}

We will assume that the marginal distribution $F$ is regular varying with continuous density $f$ that is also regularly varying i.e.

$$
\lim _{u \rightarrow \infty} \frac{\bar{F}(x u)}{\bar{F}(u)}=x^{-\alpha} \text { and } \quad \lim _{u \rightarrow \infty} \frac{f(x u)}{f(u)}=x^{-\alpha-1} .
$$

An introduction to regularly varying functions can be found in Bingham et al. (1989). Note that the assumption that $F$ is continuously differentiable is a little stronger than the assumption in the independent case (c.f. Barbe and McCormick 2009), since we assume differentiability for all values of $x$. We need this condition since unlike in the independent case also the left tail of the marginal distribution can have an influence on the asymptotic behavior (c.f. Proposition 4.3 below).

To assess the dependence between the random variables $X_{1}, \ldots, X_{n}$, we assume that we know its multivariate distribution function or equivalently, its copula $C$ defined through

$$
\mathbb{P}\left(X_{1} \leq x_{1}, \ldots, X_{n} \leq x_{n}\right)=C\left(F\left(x_{1}\right), \ldots, F\left(x_{n}\right)\right)
$$

To shorten notation for every $1 \leq m \leq n$ let $\mathbf{y}_{-m}:=\left(y_{1}, \ldots, y_{m-1}, y_{m+1}, \ldots y_{n}\right)$ and for functions

$$
C\left(F\left(\mathbf{y}_{-m}\right), F(y)\right):=C\left(F\left(y_{1}\right), \ldots, F\left(y_{m-1}\right), F(y), F\left(y_{m+1}\right), \ldots, F\left(y_{n}\right)\right)
$$

and $f\left(\mathbf{y}_{-m}\right):=\prod_{i \neq m} f\left(y_{i}\right)$. For partial derivatives of $C$ with respect to the $m$-th variable we write $C_{m}$ hence $C_{1 \ldots n}$ denotes the density of the copula (which we assume that exists). We will denote the density of the marginal copula of the $i$-th and $j$-th $(i \neq j)$ variable with

$$
C_{i j}^{m}\left(x_{i}, x_{j}\right)=\int_{0}^{1} \cdots \int_{0}^{1} C_{1 \cdots n}\left(x_{1}, \ldots, x_{n}\right) \mathrm{d} \mathbf{x}_{-\{i, j\}}
$$

Conditional probabilities can be expressed through the copula by

$$
\begin{aligned}
\mathbb{P}\left(X_{1}\right. & \left.\leq x_{1}, \ldots X_{m-1} \leq x_{m-1}, X_{m+1} \leq x_{m+1}, \ldots, X_{n-1} \leq x_{n-1} \mid X_{m}=u\right) \\
& =\left.\frac{\partial}{\partial y} C\left(F\left(\mathbf{x}_{-m}\right), y\right)\right|_{y=F(u)}=C_{m}\left(F\left(\mathbf{x}_{-m}\right), F(u)\right) .
\end{aligned}
$$

In the case that $C_{1 \ldots n}$ and the density $f$ are continuous function one can easily show that for every $u$ this defines a $(n-1)$-dimensional distribution. When ever we will 
refer to conditional distributions in this paper we mean the version defined through Eq. 4. Related to the conditional distributions is the function

$$
h_{i, j}(s, t):=\int_{1-s}^{1} C_{i j}^{m}(x,(1-t)) \mathrm{d} x .
$$

Note that

$$
h_{i, j}(\bar{F}(\delta u), \bar{F}(y u))=\mathbb{P}\left(X_{i}>\delta u \mid X_{j}=u y\right)
$$

If Eq. 3 holds then we have to assume that the probability that two variables $X_{i}$ and $X_{j}$ are large in common or that one variable $X_{j}$ is much larger then $u$ is asymptotically negligible for the second order approximation. These conditions correspond in the independent case to the condition that $\alpha>1$. These assumptions can be expressed in terms of conditional distributions, or equivalently in terms of the functions $h_{i, j}$.

Assumption 2.1 There exits a $\hat{c}_{1}>0,0<\epsilon_{1}<1, \epsilon_{2}>0$ such that for all $i \neq j$

$$
\lim _{a \rightarrow 0} \sup _{\epsilon_{1}<y<\left(1+\epsilon_{2}\right)} \frac{h_{i, j}(a, y a)}{h(i, j(a, a)} \leq \hat{c}_{1}
$$

Assumption 2.2 For some $\epsilon_{0}>0, \gamma_{1}>\gamma_{2}>\frac{1}{\alpha}, \hat{c}_{2}>0, \hat{c}_{3}>0$ and all $\left(1+\epsilon_{0}\right)$ $(2(n-1))^{\alpha}<\delta<M$ uniformly for $y \in[\delta, M]$,

$$
\hat{c}_{2}(y / \delta)^{\gamma_{1}} \leq \liminf _{a \rightarrow 0} \frac{h_{i, j}(y a, a)}{h_{i, j}(\delta a, a)} \leq \limsup _{a \rightarrow 0} \frac{h_{i, j}(y a, a)}{h_{i, j}(\delta a, a)} \leq \hat{c}_{3}(y / \delta)^{\gamma_{2}}, \quad i \neq j .
$$

Further for $\epsilon_{0}>0, \gamma_{3}>\frac{1}{\alpha}, \hat{c}_{4}>0$ and $\delta=\left(1+\epsilon_{0}\right)(2(n-1))^{\alpha}$, uniformly for $y \in(0, \delta]$

$$
\limsup _{a \rightarrow 0} \frac{h_{i, j}(y a, a)}{h_{i, j}(\delta a, a)} \leq \hat{c}_{4} y^{\gamma_{3}}, \quad i \neq j
$$

The upper tail-dependence coefficients are specified by (see e.g. Coles et al. 1999)

$$
\lambda_{i, j}:=\lim _{u \rightarrow \infty} \frac{\mathbb{P}\left(X_{i}>u, X_{j}>u\right)}{\mathbb{P}\left(X_{i}>u\right)} \quad \text { and } \quad \rho_{i, j}=\lim _{u \rightarrow \infty} \frac{2 \log \left(\mathbb{P}\left(X_{i}>u\right)\right)}{\log \left(\mathbb{P}\left(X_{i}>u, X_{j}>u\right)\right.}-1
$$

Note that $0 \leq \lambda_{i, j} \leq 1$ and $-1 \leq \rho_{i, j} \leq 1$. Further if $\lambda_{i, j}>0$ then $\rho_{i, j}=1$. In this paper we will assume that $\lambda_{i, j}=0$ for all $i \neq j$. Then it is well known (see e.g. Albrecher et al. 2006 or Davis and Resnick 1996) that for $X_{1}, \ldots, X_{n}$ with common regularly varying marginal distribution (1) holds. If $\rho_{i, j}>-1$ exists, then

$$
\mathbb{P}\left(X_{i}>u, X_{j}>u\right)=p_{i, j}(u) u^{-\frac{2 \alpha}{1+\rho_{i, j}}},
$$


where $|p(u)|$ can be bounded by a slowly varying function. Hence we will assume that $p_{i, j}(u)$ is slowly varying, or equivalently

\section{Assumption 2.3}

$$
\lim _{u \rightarrow \infty} \frac{\mathbb{P}\left(X_{i}>x u, X_{j}>x u\right)}{\mathbb{P}\left(X_{i}>u, X_{j}>u\right)}=x^{-\frac{2 \alpha}{1-\rho_{i, j}}}, \quad x>0 .
$$

A refinement of the tail-dependence coefficients $\left(\lambda_{i, j}\right.$ and $\left.\rho_{i, j}\right)$ is given by second order regular variation (c.f. de Haan and de Ronde 1998; de Haan and Resnick 1993; Resnick 2002), which in the case of $\lambda_{i, j}=0$ is defined through

$$
\lim _{u \rightarrow \infty} \frac{\frac{\mathbb{P}\left(\frac{\mathbf{x}}{u} \in[0, \mathbf{x}]^{c}\right)}{\mathbb{P}\left(X_{1}>u\right)}-\left(\sum_{i=1}^{n} x_{i}^{-\alpha}\right)}{A(b(t))}=\psi\left(x_{1}, \ldots, x_{n}\right)
$$

where $A(u)$ is regularly varying function and the limit exists locally uniform for all $0<x_{i} \leq \infty$. In the case of independent random variables second order regular variation can be used to get higher order asymptotic approximation (c.f. Geluk 1992; Geluk et al. 1997). Note that Eq. 5 implies a second order condition on the marginal distribution $F$ which we don't assume in this paper. On the other hand we will see from Propositions 4.1 and 4.3 that the second order asymptotic behavior can be influenced by the left tail of the marginal distribution. Hence one needs further conditions on the dependence structure to get second order asymptotic approximations.

\section{Asymptotic results}

For our main result, we will need the following additional conditions

Assumption 3.1 For every $i \neq j \mathbb{E}\left[X_{i} \mid X_{j}=u\right]$ is of consistent variation. i.e.

$$
\begin{aligned}
& \lim _{\epsilon \rightarrow 0} \limsup _{a \rightarrow 0} \frac{\int_{0}^{1} F^{-1}(x) C_{i j}^{m}(x, 1-(1+\epsilon) a) \mathrm{d} x}{\int_{0}^{1} F^{-1}(x) C_{i j}^{m}(x, 1-a) \mathrm{d} x} \\
& \quad=\lim _{\epsilon \rightarrow 0} \liminf _{a \rightarrow 0} \frac{\int_{0}^{1} F^{-1}(x) C_{i j}^{m}(x, 1-(1+\epsilon) a) \mathrm{d} x}{\int_{0}^{1} F^{-1}(x) C_{i j}^{m}(x, 1-a) \mathrm{d} x}=1
\end{aligned}
$$

Assumption 3.2 For every $0<\epsilon \leq 1 / 2$, there exist sets $A(\epsilon)=A(\epsilon, m)$ such that uniformly for $\left\{0<u x<\sum_{i \neq m} y_{i}, \max _{i \neq m} y_{i}<\epsilon u\right\} \cap\left\{\mathbf{y}_{-m} \in A(\epsilon)\right\}$

$$
\begin{aligned}
& \left(1-o_{\epsilon}(1)\right) C_{1 \cdots n}\left(F\left(\mathbf{y}_{-m}\right), F(u)\right) \lesssim C_{1 \cdots n}\left(F\left(\mathbf{y}_{-m}\right), F(u(1-x))\right) \\
& \lesssim\left(1+o_{\epsilon}(1)\right) C_{1 \cdots n}\left(F\left(\mathbf{y}_{-m}\right), F(u(1-(n-1) \epsilon))\right)
\end{aligned}
$$


and uniformly on $\left\{0<u x<\sum_{i \neq m} y_{i}, \max _{i \neq m} y_{i}<\epsilon u\right\} \cap\left\{\mathbf{y}_{-m} \in A(\epsilon)^{c}\right\}$

$$
\begin{gathered}
\left(1+o_{\epsilon}(1)\right) C_{1 \cdots n}\left(F\left(\mathbf{y}_{-m}\right), F(u)\right) \gtrsim C_{1 \cdots n}\left(F\left(\mathbf{y}_{-m}\right), F(u(1-x))\right) \\
\gtrsim\left(1-o_{\epsilon}(1)\right) C_{1 \cdots n}\left(F\left(\mathbf{y}_{-m}\right), F(u(1-(n-1) \epsilon))\right),
\end{gathered}
$$

where $o_{\epsilon}(1)$ is a function that approaches zero as $\epsilon \rightarrow 0$. Further we have to assume that for every $i \neq m, A(\epsilon)$ fulfills

$$
\mathbb{E}\left[X_{i} 1_{\{A(\epsilon)\}} \mid X_{n}=u\right] \sim\left(1+o_{\epsilon}(1)\right) \mathbb{E}\left[X_{i} 1_{\{A(\epsilon)\}} \mid X_{n}=u(1-(n-1) \epsilon)\right] .
$$

Remark 3.1 If for the set $A(\epsilon)$ in Assumption 3.2 it holds for all $\epsilon>0$

$$
\mathbb{E}\left[X_{i} 1_{\{A(\epsilon)\}} \mid X_{n}=u\right]=o\left(\mathbb{E}\left[X_{i} \mid X_{n}=u\right]\right)
$$

Then Eq. 6 is interpreted as

$$
\mathbb{E}\left[X_{i} 1_{\{A(\epsilon)\}} \mid X_{n}=u(1-(n-1) \epsilon)\right]=o\left(\mathbb{E}\left[X_{i} \mid X_{n}=u\right]\right)
$$

Remark 3.2 Note that for the copulas presented in Sections 5 and 6 we only need that the marginal distribution is regularly varying to show that Assumptions 3.1 and 3.2 are fulfilled.

Theorem 3.1 Let $X_{1}, \ldots, X_{n}$ be dependent random variables with copula $C$ that has a continuous density $C_{1 \cdots n}$ and a common marginal distribution function $F$ which is continuously differentiable with regularly varying density $f$ with index $-\alpha-1$. Further assume that Assumptions 2.1-2.3, 3.1 and 3.2 are fulfilled. Then

$$
\mathbb{P}\left(S_{n}>u\right)=n \bar{F}(u)+(1+o(1)) f(u) \sum_{i=1}^{n} \sum_{j=1, j \neq i}^{n} \mathbb{E}\left[X_{j} \mid X_{i}=u\right]
$$

Corollary 3.2 Assume that the conditions of Theorem 3.1 hold then

$$
\begin{aligned}
& \left|\mathbb{P}\left(S_{1}>u\right)-\sum_{i=1}^{n} \mathbb{P}\left(X_{i}>u-\mathbb{E}\left[S_{n}-X_{i} \mid X_{i}=u\right]\right)\right| \\
& \quad=o(1) f(u) \sum_{i=1}^{n} \mathbb{E}\left[S_{n}-X_{i} \mid X_{i}=u\right]
\end{aligned}
$$




\section{Multivariate Gaussian copula}

As a first example we consider the Gaussian copula. In the two-dimensional case, the density of the Gaussian copula is given by

$$
C_{x, y}(x, y)=\frac{1}{\sqrt{1-\rho^{2}}} \exp \left(-\frac{\rho^{2} \Phi^{-1}(x)^{2}+\rho^{2} \Phi^{-1}(y)^{2}-2 \rho \Phi^{-1}(x) \Phi^{-1}(y)}{2\left(1-\rho^{2}\right)}\right),
$$

where

$$
\phi(x)=\frac{1}{\sqrt{2 \pi}} e^{-\frac{x^{2}}{2}} \text { and } \Phi(x)=\int_{-\infty}^{x} \phi(x) \mathrm{d} x .
$$

Let $\mathbf{x}=\left(x_{1}, \ldots, x_{n}\right)$. The density of the $n$-dimensional Gaussian Copula is given by

$$
C_{1 \cdots n}(\mathbf{x})=\frac{1}{\sqrt{(2 \pi)^{n} \operatorname{det}(\Sigma)}} \frac{\exp \left(-\frac{1}{2} \Phi^{-1}(\mathbf{x})^{T} \Sigma^{-1} \Phi^{-1}(\mathbf{x})\right)}{\prod_{i=1}^{n} \phi\left(\Phi^{-1}\left(x_{i}\right)\right)}
$$

where $\Sigma$ is the correlation matrix of a Gaussian random vector.

Proposition 4.1 Assume that $X_{1}, \ldots, X_{n}$ follow a Gaussian copula with correlation matrix $\Sigma$ and entries $-1<\rho_{i, j}<1$. If the marginal distribution $F$ fulfills the Assumption of Theorem 3.1 with $\alpha>\max _{i \neq j}\left(\left(1+\rho_{i, j}\right) \vee\left(1-\rho_{i, j}^{2}\right)\right)$ and one of the following conditions is fulfilled

(I) For all $i \neq j, \rho_{i, j}>0$

(II) $F(x)$ has a left endpoint $x_{F}>0$

(III) $F(x)$ is regularly varying at zero with index $\tau>0$,

then Assumptions 2.1-2.3, 3.1 and 3.2 are fulfilled.

Proposition 4.2 Let $X_{1}, X_{2}$ be two dependent random variables with common marginal distribution $F$, where the dependence is given by a Gaussian Copula with correlation $\rho>0$. If for $x_{0}<x<1$

$$
F^{-1}(1-x)=\kappa x^{-1 / \alpha}+r(x),
$$

with $r(x) \leq K x^{-\beta}, \beta<1 / \alpha$ and $\kappa>0$, then

$$
\begin{aligned}
\mathbb{E}\left[X_{2} \mid X_{1}=u\right] \sim & \kappa\left(\frac{\rho \alpha}{\alpha+\rho^{2}-1}\right)^{1 / \alpha} \\
& \times \sqrt{\frac{\alpha}{\alpha+\rho^{2}-1}}(-4 \pi \log (\bar{F}(u)))^{\frac{1}{2 \alpha}-\frac{\rho^{2}}{2\left(\alpha+\rho^{2}-1\right)}} \bar{F}(u)^{-\frac{\rho^{2}}{\alpha+\rho^{2}-1}} .
\end{aligned}
$$


Proposition 4.3 Let $X_{1}, X_{2}$ be two dependent random variables with common marginal distribution $F$ that is regularly varying with index $\alpha$, where the dependence is given by a Gaussian copula with correlation $\rho<0$. If the left endpoint is $x_{F}>0$ then

$$
\lim _{u \rightarrow \infty} \mathbb{E}\left[X_{2} \mid X_{1}=u\right]=x_{F}
$$

If $x_{F}=0$ and, as $x \rightarrow 0$,

$$
F^{-1}(x)=\kappa x^{\tau}+r_{1}(x)
$$

with $r_{1}(x) \leq K x^{\beta_{1}}, \tau<\beta$ and $\kappa>0$ then

$$
\begin{aligned}
\mathbb{E}\left[X_{2} \mid X_{1}=u\right] \sim & \kappa\left(\frac{-\rho}{1+\tau\left(1-\rho^{2}\right)}\right)^{-\tau} \sqrt{\frac{1}{1+\tau\left(1-\rho^{2}\right)}} \\
& \times(-4 \pi \log (\bar{F}(u)))^{-\frac{\tau}{2}+\frac{\tau \rho^{2}}{2\left(1+\tau\left(1-\rho^{2}\right)\right)} \bar{F}(u)^{\frac{\tau \rho^{2}}{1+\tau\left(1-\rho^{2}\right)}}}
\end{aligned}
$$

\section{Archimedean copulas}

Consider now Archimedean copulas (c.f. Nelsen 2006) with generator $\varphi(x),[0,1] \rightarrow$ $[\infty, 0]$, where $\varphi(x)$ is strictly decreasing. The Archimedean Copula is then defined by

$$
C\left(x_{1}, \ldots, x_{n}\right)=\varphi^{-1}\left(\sum_{i=1}^{n} \varphi\left(x_{i}\right)\right)
$$

To ensure that $C$ is a copula for all $n$, we further assume that $\varphi$ is strict (i.e. $\varphi(0)=\infty)$ and $\varphi^{-1}$ is completely monotone, hence $\varphi^{-1}$ has derivatives of all orders $\left(\varphi^{-1}\right)^{(k)}(x)$ that alternate in sign. Further there exists a positive random variable $Z$ with

$$
\left(\varphi^{-1}\right)^{(k)}(x)=\mathbb{E}\left[Z^{k} e^{-x Z}\right], \quad x \in[0, \infty) .
$$

The tail-dependence coefficient is then given by (cf. Nelsen 2006, Corollary 5.4.3)

$$
\lambda=2-\lim _{x \rightarrow 0^{+}} \frac{1-\varphi^{-1}(2 x)}{1-\varphi^{-1}(x)} .
$$

Further if $\lambda=0$ then for all $n>0$ it holds that

$$
\lim _{u \rightarrow \infty} \frac{\mathbb{P}\left(X_{1}>u, \ldots, X_{n}>u\right)}{\mathbb{P}\left(X_{1}>u\right)}=0 .
$$


hence the inclusion-exclusion principle implies

$$
\begin{aligned}
\lim _{x \rightarrow 0^{+}} \frac{1-\varphi^{-1}(n x)}{1-\varphi^{-1}(x)} & =\lim _{u \rightarrow \infty} \frac{1-\varphi^{-1}(n \varphi(F(x)))}{1-\varphi^{-1}(\varphi(F(x)))} \\
& =\lim _{u \rightarrow \infty} \frac{1-\mathbb{P}\left(X_{1} \leq u, \ldots, X_{n} \leq u\right)}{\mathbb{P}\left(X_{1}>u\right)}=n
\end{aligned}
$$

Consequently if $\lambda=0$ then $1-\varphi^{-1}(x)$ is regularly varying at 0 with index 1 . To prove Assumptions 2.1-2.3, 3.1 and 3.2, we will need some further conditions on $\varphi(x)$.

Proposition 5.1 Let $X_{1}, \ldots, X_{n}$ be dependent random variables with marginal distribution $F$ that fulfill the condition of Theorem 3.1 and copula $C$ which is Archimedean with strict generator $\varphi$ that is completely monotone. Further assume

(a) $\varphi^{-1}(x)=1-c x-x^{\beta} L(x), L(1 / x)$ slowly varying and $1<\beta \leq 2, c>0$,

(b) $\left(\varphi^{-1}\right)^{\prime}(x)=-c-\beta x^{\beta-1} L_{2}(x)$, where $\lim _{x \rightarrow 0} L(x) / L_{2}(x)=1$,

(c) if $\beta=2$ we further assume that $0<\left(\varphi^{-1}\right)^{\prime \prime}(0)<\infty$,

(d) $\left(\varphi^{-1}\right)^{\prime \prime}(x)$ is regularly varying at 0 ,

(e) $\alpha>(1+\rho) /(1-\rho)=1 /(\beta-1)$.

Then Assumptions 2.1-2.3, 3.1 and 3.2 are fulfilled. Further

$$
\lim _{u \rightarrow \infty} \mathbb{E}\left[X_{1} \mid X_{2}=u\right]=-\frac{1}{c} \int_{0}^{\infty} x\left(\varphi^{-1}\right)^{\prime \prime}(\varphi(F(x)))\left(\varphi^{\prime}(F(x))\right) f(x) \mathrm{d} x<\infty .
$$

Remark 5.1 The assumptions (a)-(d) are fulfilled for the families of copulas provided in Nelsen (2006, Table 4.1) for which the inverse of the generator is completely monotone and $\lambda=0$. These are the families $1(\theta \geq 0), 3(\theta \geq 0), 5(\theta>0)$, $13(\theta>1), 17(\theta>-1), 19$ and 20. Further for all of these copulas we have $\beta=2$.

\section{Copulas with bounded densities}

In this section we consider two-dimensional copulas which have a density that can be bounded from above and below; examples are the Placket family (c.f. Nelsen 2006)

$$
\begin{aligned}
& C\left(x_{1}, x_{2}\right) \\
& :=\frac{\left(1+(\theta-1)\left(x_{1}+x_{2}\right)\right)-\sqrt{\left(1+(\theta-1)\left(x_{1}+x_{2}\right)\right)^{2}-4 x_{1} x_{2} \theta(\theta-1)}}{2(\theta-1)}, \quad \theta>0,
\end{aligned}
$$

the Ali-Mikhail-Haq family with

$$
C\left(x_{1}, x_{2}\right):=\frac{x_{1} x_{2}}{1-\theta\left(1-x_{1}\right)\left(1-x_{2}\right)}, \quad-1<\theta<1
$$


Fig. 1 A plot of relative errors and convergence rate for a Gaussian copula with $\rho=0.9$ and Pareto marginals with $\alpha=2$.

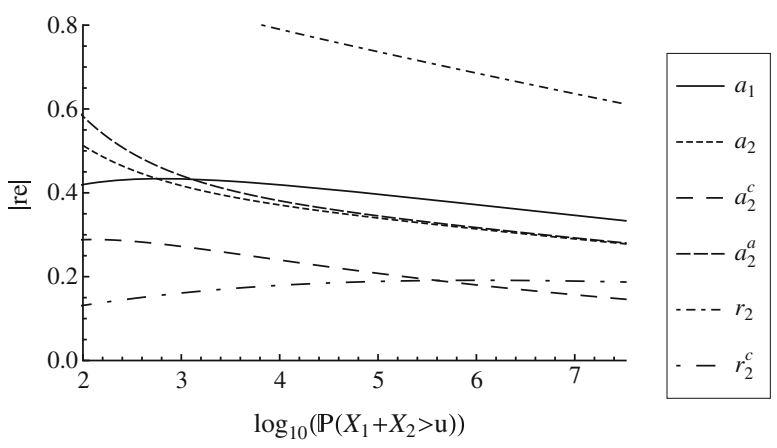

and the Farlie-Gumbel-Morgen stern family of copulas with

$$
C\left(x_{1}, x_{2}\right)=x_{1} x_{2}+\theta x_{1} x_{2}\left(1-x_{1}\right)\left(1-x_{2}\right), \quad-1 \leq \theta<1 .
$$

Proposition 6.1 Assume that $X_{1}, \ldots, X_{n}$ are dependent according to a copula $C$ with continous density $C_{1 \ldots n}$. If the marginal distribution $F$ fulfills the conditions of Theorem 3.1 with $\alpha>1$ and there exits constants $m<M$ with

$$
m \leq \inf _{0 \leq x_{i} \leq 1} C_{1 \cdots n}\left(x_{1}, \ldots, x_{n}\right) \leq \sup _{0 \leq x_{i} \leq 1} C_{1 \cdots n}\left(x_{1}, \ldots, x_{n}\right) \leq M
$$

then Assumptions 2.1-2.3, 3.1 and 3.2 are fulfilled. Further

$$
\lim _{u \rightarrow \infty} \mathbb{E}\left[X_{1} \mid X_{2}=u\right]=\int_{0}^{1} F^{-1}(x) C_{i j}^{m}(x, 1) \mathrm{d} x<\infty .
$$

\section{Numerical examples}

In this section we provide numerical examples for the derived asymptotic approximations. To that end we will use a two-dimensional Gaussian copula with $\rho \in$

Fig. 2 A plot of relative errors and convergence rate for a Gaussian copula with $\rho=0.5$ and Pareto marginals with $\alpha=2$.

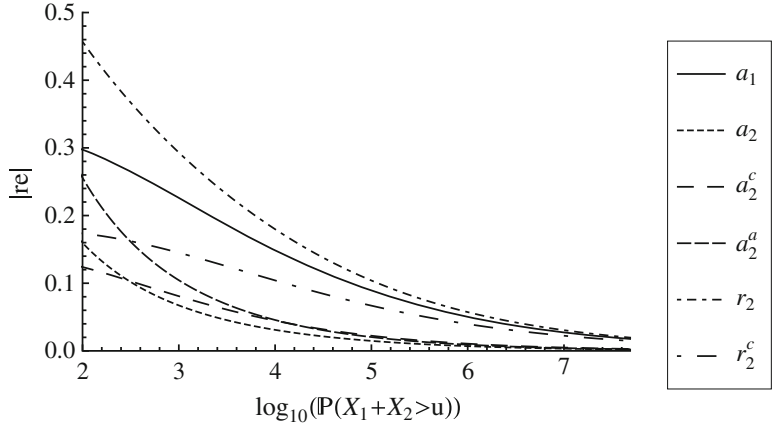


Fig. 3 A plot of relative errors and for a Gaussian copula with $\rho=-0.5$ and Pareto marginals with $\alpha=2$.

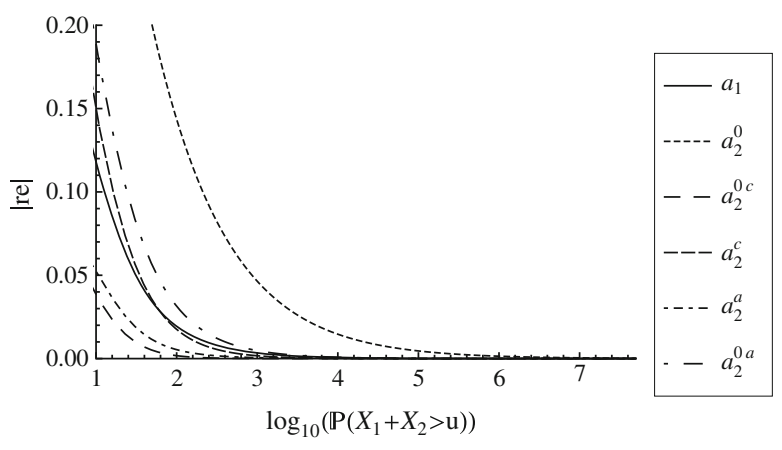

$\{0.9,0.5,-0.5\}$. For the marginal distribution we will use a Pareto distribution with tail $\bar{F}(x)=(1+x)^{-\alpha}$ and $\alpha=2$. For $\rho=-1 / 2$, we also use a shifted Pareto distribution as marginal distribution with $\bar{F}(x)=x^{-\alpha}$ and again $\alpha=2$. At first we discuss the case $\rho=0.9$. Figure 1 shows a plot of the absolute value of the relative error of the first order asymptotic approximation $\left(a_{1}\right)$ and the refined asymptotic approximation of Eq. $3\left(a_{2}\right)$. Further we used the approximation $\mathbb{E}\left[X_{2} \mid X_{1}=u\right]$ replaced by the asymptotic provided in Proposition $4.2\left(a_{2}^{a}\right)$. Since in the proof of Theorem 3.1 we condition on $X_{i} \leq \delta / u$ with $\delta<u /(2(n-2)$ we also provided an approximation with $\mathbb{E}\left[X_{2} \mid X_{1}=u\right]$ replaced by $\mathbb{E}\left[X_{2} 1_{\left\{X_{2}<u / 2\right\}} \mid X_{1}=u\right]\left(a_{2}^{c}\right)$. The $x$-axis of the plot is $-\log _{10}\left(\mathbb{P}\left(X_{1}+X_{2}>u\right)\right)$. In Fig. 1 we can see that the approximation $2 \bar{F}(u)$ to $\mathbb{P}\left(X_{1}+X_{2}>u\right)$ is rather slow, a fact that is also observed in Mitra and Resnick (2009) where lognormal marginals are considered. Further we observe that the second order asymptotics $a_{2}$ and $a_{2}^{a}$ behave quite similarly, but only improve slightly over the first order asymptotics. The asymptotic approximation $a_{2}^{c}$ is significantly better than the others, but still not satisfactorily good. Further if we look at the rate of convergence, we see that the error term used in $a_{2}, r_{2}$ overestimates the error while $r_{2}^{c}$ underestimates the error. Both of these error terms are far away from the real error. However, they provide the correct order for the error. Figure 2 gives basically the same conclusions as Fig. 1. The main difference is that in this case the asymptotic approximation is significantly better. Depending on the threshold $u$ and the quality criteria one is using, it can be considered acceptable. In Fig. 3 we see the corresponding plot for $\rho=-0.5$. As expected from our theoretical findings the error of the asymptotic approximation for $\bar{F}(x)=x^{-\alpha}\left(a_{1}^{0}\right)$ is significantly bigger than in the case of $\bar{F}(x)=(1+x)^{-\alpha}$. The same is true for the second order approximation $a_{2}^{0 c}$ and $a_{2}^{0 a}$ distribution which are defined analogously to $a_{2}^{c}$ respectively $a_{2}^{a}$ only for $\bar{F}(x)=x^{-\alpha}$ instead of $\bar{F}(x)=(1+x)^{-\alpha}$

\section{Conclusion}

In this paper we considered dependent regularly varying random variables which are asymptotically independent. In this case it is known that the sum behaves asymptotically like in the independent case. Under some conditions on the copula we showed 
that the convergence rate is of a similar form as in the independent case. Further these formulas were used to improve the approximation that is given by the first-order asymptotic.

Acknowledgments I would like to thank Hansjörg Albrecher, Enkelejd Hashorva and two anonymous referees for useful remarks on the paper.

\section{Appendix A: Proofs of Section 3}

In this section we denote by $d_{i}$ some constants and we denote with

$$
\begin{aligned}
a_{i}(u) & :=\int_{0}^{\frac{u}{2(n-1)}} x C_{i n}^{m}(F(x), F(u)) f(x) f(u) \mathrm{d} x \\
& =f(u) \mathbb{E}\left[X_{i} 1_{\left\{X_{i} \leq \frac{u}{2(n-1)}\right\}} \mid X_{n}=u\right] .
\end{aligned}
$$

Note that from Assumption 2.1 and Potter bounds (e.g. Bingham et al. 1989) it follows that there exits a $c_{1}>0, M>1$ such that for all $u>u_{0}$, all $1<y<M$ and all $i \neq j$

$$
\frac{\mathbb{P}\left(X_{i}>u \mid X_{j}=y u\right)}{\mathbb{P}\left(X_{i}>u \mid X_{j}=u\right)} \leq c_{1} .
$$

Similarly from Assumption 2.2 it follows that For some $\beta_{1}>\beta_{2}>1, c_{2}>0, c_{3}>0$ and all $0<\delta<1 /(2(n-1)), 0<\epsilon<\delta, i \neq j$ uniformly for $y \in[\epsilon, \delta]$,

$$
\begin{aligned}
c_{2}(y / \delta)^{-\beta_{1}} & \leq \liminf _{u \rightarrow \infty} \frac{\mathbb{P}\left(X_{i}>y u \mid X_{j}=u\right)}{\mathbb{P}\left(X_{i}>\delta u \mid X_{j}=u\right)} \\
& \leq \limsup _{u \rightarrow \infty} \frac{\mathbb{P}\left(X_{i}>y u \mid X_{j}=u\right)}{\mathbb{P}\left(X_{i}>\delta u \mid X_{j}=u\right)} \leq c_{3}(y / \delta)^{-\beta_{2}}
\end{aligned}
$$

and for some $\beta_{3}>1, c_{4}>0$ and uniformly for $y \in[1 /(2(n-1)), \infty]$,

$$
\limsup _{u \rightarrow \infty} \frac{\mathbb{P}\left(X_{i}>y u \mid X_{j}=u\right)}{\mathbb{P}\left(X_{i}>\delta u \mid X_{j}=u\right)} \leq c_{4} y^{-\beta_{3}}, \quad i \neq j .
$$

For the proof of Theorem 3.1 we need the following three lemmas.

Lemma A.1 Under the assumptions of Theorem 3.1 for every $0<\delta<1 /(2(n-1))$ and $1 \leq i \leq n-1$

$$
\begin{aligned}
& \int_{[0, \delta u]^{n-1}} x_{i} C_{1 \cdots n}\left(F\left(\mathbf{x}_{-n}\right), F(u)\right) f\left(\mathbf{x}_{-n}\right) f(u) \mathrm{d} \mathbf{x}_{-n} \\
& \sim a_{i}(u)+o\left(\sum_{j \notin\{i, n\}} a_{j}(u)\right)
\end{aligned}
$$


and

$$
\begin{aligned}
& \mathbb{P}\left(X_{i}>u, X_{n}>u\right) \\
& \quad=o\left(\int_{0}^{\frac{u}{2(n-1)}} x\left[C_{i n}^{m}(F(x), F(u))+C_{i n}^{m}(F(u), F(x))\right] f(x) f(u) \mathrm{d} x\right) .
\end{aligned}
$$

Proof of Lemma A.1 W.1.o.g. we choose $i=n-1$. We have that

$$
\begin{aligned}
f(u) & \int_{[0, \delta u]^{n-1}} x_{n-1} C_{1 \cdots n}\left(F\left(\mathbf{x}_{-n}\right), F(u)\right) f\left(\mathbf{x}_{-n}\right) \mathrm{d} \mathbf{x}_{-n} \\
= & f(u) \int_{0}^{\delta u} x C_{(n-1) n}(F(x), F(u)) f(x) \mathrm{d} x \\
& -\sum_{j=1}^{d} f(u) \int_{[0, \infty)^{n-2-j} \times(\delta u, \infty) \times[0, \infty)^{j-1} \times[0, \delta u]} x_{n-1} C_{1 \cdots n}\left(F\left(\mathbf{x}_{-n}\right),\right. \\
& F(u)) f\left(\mathbf{x}_{-n}\right) \mathrm{d} \mathbf{x}_{-n} .
\end{aligned}
$$

At first note that

$$
\begin{aligned}
f(u) & \int_{0}^{\delta u} x C_{(n-1) n}(F(x), F(u)) f(x) \mathrm{d} x \\
= & f(u) \int_{0}^{\delta u} \int_{y}^{\infty} C_{(n-1) n}(F(x), F(u)) f(x) \mathrm{d} x \mathrm{~d} y \\
& -\delta u f(u) \int_{\delta u}^{\infty} C_{(n-1) n}(F(x), F(u)) f(x) \mathrm{d} x .
\end{aligned}
$$

From Eq. 9 we get that there exists $\beta_{1}>0$ and $d_{1}>0$ such that for every $0<\epsilon<\delta$

$$
\begin{aligned}
& f(u) \int_{0}^{\delta u} \int_{y}^{\infty} C_{(n-1) n}(F(x), F(u)) f(x) \mathrm{d} x \mathrm{~d} y \\
& \quad \geq u f(u) \int_{\epsilon}^{\delta} \int_{y u}^{\infty} C_{(n-1) n}(F(x), F(u)) f(x) \mathrm{d} x \mathrm{~d} y \\
& \quad \geq d_{1} u f(u) \int_{\epsilon}^{\delta}(y / \delta)^{-\beta_{1}} \mathrm{~d} y \int_{\delta u}^{\infty} C_{(n-1) n}(F(x), F(u)) f(x) \mathrm{d} x .
\end{aligned}
$$

With $\epsilon \rightarrow 0$ it follows that

$$
\begin{aligned}
f(u) & \int_{0}^{\delta u} x C_{(n-1) n}(F(x), F(u)) f(x) \mathrm{d} x \\
& \sim f(u) \int_{0}^{\delta u} \int_{y}^{\infty} C_{(n-1) n}(F(x), F(u)) f(x) \mathrm{d} x \mathrm{~d} y
\end{aligned}
$$


and

$$
\begin{aligned}
& \delta u f(u) \int_{\delta u}^{\infty} C_{(n-1) n}(F(x), F(u)) f(x) \mathrm{d} x \\
& \quad=o\left(f(u) \int_{0}^{\delta u} x C_{(n-1) n}(F(x), F(u)) f(x) \mathrm{d} x\right) .
\end{aligned}
$$

For $1 \leq j \leq n-2$ we get

$$
\begin{aligned}
f(u) & \int_{[0, \infty)^{n-2-j} \times(\delta u, \infty) \times[0, \infty)^{j-1} \times[0, \delta u]} x_{n-1} C_{1 \cdots n}\left(F\left(\mathbf{x}_{-n}\right), F(u)\right) f\left(\mathbf{x}_{-n}\right) \mathrm{d} \mathbf{x}_{-n} \\
\leq & \delta u f(u) \int_{\delta u}^{\infty} C_{j n}^{m}(F(x), F(u)) f(x) \mathrm{d} x \\
= & o\left(f(u) \int_{0}^{\delta u} x C_{j n}^{m}(F(x), F(u)) f(x) \mathrm{d} x\right) .
\end{aligned}
$$

With Eq. 9 we get for $\beta_{2}>1$ and $d_{2}>0$

$$
\begin{aligned}
& f(u) \int_{\delta u}^{\frac{u}{2(n-1)}} \int_{y}^{\infty} C_{(n-1) n}(F(x), F(u)) f(x) \mathrm{d} x \mathrm{~d} y \\
& \leq d_{2} u f(u) \int_{\delta}^{\frac{1}{2(n-1)}}(y / \delta)^{-\beta_{2}} \mathrm{~d} y \int_{\delta u}^{\infty} C_{j n}^{m}(F(x), F(u)) f(x) \mathrm{d} x,
\end{aligned}
$$

hence Eq. 11 follows.

To prove Eq. 12 note that by Assumption 2.3 for $M>0$

$$
\begin{gathered}
\mathbb{P}\left(X_{n-1}>u, X_{n}>u\right)-\mathbb{P}\left(X_{n-1}>M u, X_{n}>M u\right) \\
\sim\left(1-M^{-\frac{2 \alpha}{1-\rho_{n-1, n}}}\right) \mathbb{P}\left(X_{n-1}>u, X_{n}>u\right) .
\end{gathered}
$$

Further

$$
\begin{aligned}
& \mathbb{P}\left(X_{n-1}>u, X_{n}>u\right)-\mathbb{P}\left(X_{n-1}>M u, X_{n}>M u\right) \\
& \leq \int_{u}^{M u} \int_{u}^{\infty} C_{(n-1) n}\left(F\left(x_{n-1}\right), F\left(x_{n}\right)\right) f\left(x_{n-1}\right) f\left(x_{n}\right) \mathrm{d} x_{n-1} \mathrm{~d} x_{n} \\
& \quad+\int_{u}^{\infty} \int_{u}^{M u} C_{(n-1) n}\left(F\left(x_{n-1}\right), F\left(x_{n}\right)\right) f\left(x_{n-1}\right) f\left(x_{n}\right) \mathrm{d} x_{n-1} \mathrm{~d} x_{n} .
\end{aligned}
$$


By Assumption 2.1 for every $\epsilon>0$ there exits $d_{3}>0$ such that

$$
\begin{aligned}
& \int_{u}^{M u} \int_{u}^{\infty} C_{(n-1) n}\left(F\left(x_{n-1}\right), F\left(x_{n}\right)\right) f\left(x_{n-1}\right) f\left(x_{n}\right) \mathrm{d} x_{n-1} \mathrm{~d} x_{n} \\
& \quad=\int_{1}^{M} \int_{u}^{\infty} C_{(n-1) n}\left(F\left(x_{n-1}\right), F(y u)\right) f\left(x_{n-1}\right) u f(y u) \mathrm{d} x_{n-1} \mathrm{~d} y \\
& \quad \leq d_{3} u f(u) \int_{u}^{\infty} C_{(n-1) n}\left(F\left(x_{n-1}\right), F(u)\right) f\left(x_{n-1}\right) \mathrm{d} x_{n-1} \int_{1}^{M} y^{-\beta_{1}-\alpha-1} \mathrm{~d} y \\
& \quad=o\left(f(u) \int_{0}^{\delta u} x C_{(n-1) n}^{m}(F(x), F(u)) f(x) \mathrm{d} x\right) .
\end{aligned}
$$

Analogously we get

$$
\begin{aligned}
& \int_{u}^{\infty} \int_{u}^{M u} C_{(n-1) n}\left(F\left(x_{n-1}\right), F\left(x_{n}\right)\right) f\left(x_{n-1}\right) f\left(x_{n}\right) \mathrm{d} x_{n-1} \mathrm{~d} x_{n} \\
& \quad=o\left(f(u) \int_{0}^{\delta u} x C_{(n-1) n}^{m}(F(u), F(x)) f(x) \mathrm{d} x\right) .
\end{aligned}
$$

Hence Eq. 12 follows.

\section{Lemma A.2 Under the assumptions of Theorem 3.1}

$$
a_{i}(u) \sim \int_{0}^{\infty} x C_{i n}^{m}(F(x), F(u)) f(x) f(u) \mathrm{d} x
$$

Proof At first note that

$$
\begin{aligned}
& \int_{0}^{\infty} x C_{i n}^{m}(F(x), F(u)) f(x) f(u) \mathrm{d} x \\
& =\lim _{t \rightarrow \infty} \int_{0}^{t} x C_{i n}^{m}(F(x), F(u)) f(x) f(u) \mathrm{d} x \\
& =\lim _{t \rightarrow \infty}\left(f(u) \int_{0}^{t} \int_{y}^{\infty} C_{i n}^{m}(F(x), F(u)) f(x) \mathrm{d} x \mathrm{~d} y\right. \\
& \left.\quad-t f(u) \int_{t}^{\infty} C_{i n}^{m}(F(x), F(u)) f(x) \mathrm{d} x\right)
\end{aligned}
$$


By Eq. 10 there exists constants $d_{1}>0$ and $\beta>1$ such that for large $u$ and $t>u / 2$

$$
\begin{aligned}
& t f(u) \int_{t}^{\infty} C_{i n}^{m}(F(x), F(u)) f(x) \mathrm{d} x \\
& \quad \leq d_{1}\left(\frac{t}{u}\right)^{-\beta} \int_{\frac{u}{2(n-1)}}^{\infty} C_{i n}^{m}(F(x), F(u)) f(x) \mathrm{d} x
\end{aligned}
$$

which tends to 0 as $t \rightarrow \infty$. As above note that

$$
\begin{aligned}
& \int_{\frac{u}{2(n-1)}}^{\infty} f(u) \int_{y}^{\infty} C_{i n}^{m}(F(x), F(u)) f(x) \mathrm{d} x \mathrm{~d} y \\
& \quad \leq d_{1} u f(u) \int_{\frac{1}{2(n-1)}}^{\infty} y^{-\beta} \mathrm{d} y \int_{\frac{u}{2(n-1)}}^{\infty} C_{i n}^{m}(F(x), F(u)) f(x) \mathrm{d} x
\end{aligned}
$$

hence the lemma follows.

Lemma A.3 Under the conditions of Theorem 3.1 for every $u-\sum_{i=1}^{n-1} x_{i}<\xi_{\mathbf{x}_{-n}, u}<u$

$$
\begin{aligned}
& \int_{[0, u /(2(n-1))]^{n-1}} x_{i} C_{1 \cdots n}\left(F\left(\mathbf{x}_{-n}\right), F\left(\xi_{\mathbf{x}_{-n}, u}\right)\right) f\left(\mathbf{x}_{-n}\right) f\left(\xi_{\mathbf{x}_{-n}, u}\right) \mathrm{d} \mathbf{x}_{-n} \\
& \quad \sim \int_{[0, u /(2(n-1))]^{n-1}} x_{i} C_{1 \cdots n}\left(F\left(\mathbf{x}_{-n}\right), F(u)\right) f\left(\mathbf{x}_{-n}\right) f(u) \mathrm{d} \mathbf{x}_{-n}+o\left(\sum_{j \notin\{i, n\}} a_{j}(u)\right) .
\end{aligned}
$$

Proof By Assumption 3.2 it follows that there exists a constant $d_{1}$ such that for all $0<\epsilon \leq 1 /(2(n-1))$

$$
\begin{aligned}
& \int_{(\epsilon u, u /(2(n-1))]^{n-1}} x_{i} C_{1 \cdots n}\left(F\left(\mathbf{x}_{-n}\right), F\left(\xi_{\mathbf{x}_{-n}, u}\right)\right) f\left(\mathbf{x}_{-n}\right) f\left(\xi_{\mathbf{x}_{-n}, u}\right) \mathrm{d} \mathbf{x}_{-n} \\
& \lesssim d_{1} f(u) \int_{(\epsilon u, u /(2(n-1))]^{n-1} \cap A(\epsilon)} x_{i} C_{1 \cdots n}\left(F\left(\mathbf{x}_{-n}\right),\right. \\
& \quad F(u(1-(n-1) \epsilon)) f\left(\mathbf{x}_{-n}\right) \mathrm{d} \mathbf{x}_{-n} \\
& \quad+d_{1} f(u) \int_{(\epsilon u, u /(2(n-1))]^{n-1} \cap A(\epsilon)^{c}} x_{i} C_{1 \cdots n}\left(F\left(\mathbf{x}_{-n}\right), F(u)\right) f\left(\mathbf{x}_{-n}\right) \mathrm{d} \mathbf{x}_{-n} \\
& =o\left(\sum_{i=1}^{n-1} a_{i}(u)\right)
\end{aligned}
$$

where the last inequality follows from Lemmas A.1, A.2 and Assumption 3.1. 
By Assumption 3.2 it follows that for all $0<\epsilon \leq 1 /(2(n-1))$ there exists $d_{2}(\epsilon)$ with $d_{2}(\epsilon) \rightarrow 0$ as $\epsilon \rightarrow 0$ such that for all $u>u_{\epsilon}$

$$
\begin{aligned}
& \int_{[0, \epsilon u]^{n-1}} x_{i} C_{1 \cdots n}\left(F\left(\mathbf{x}_{-n}\right), F\left(\xi_{\mathbf{x}_{-n}, u}\right)\right) f\left(\mathbf{x}_{-n}\right) f\left(\xi_{\mathbf{x}_{-n}, u}\right) \mathrm{d} \mathbf{x}_{-n} \\
& \lesssim\left(1+d_{2}(\epsilon)\right) f(u) \int_{[0, \epsilon u]^{n-1} \cap A(\epsilon)} x_{i} C_{1 \cdots n}\left(F\left(\mathbf{x}_{-n}\right),\right. \\
& \quad F(u(1-(n-1) \epsilon))) f\left(\mathbf{x}_{-n}\right) \mathrm{d} \mathbf{x}_{-n} \\
& \quad+\left(1+d_{2}(\epsilon)\right) f(u) \int_{[0, \epsilon u]^{n-1} \cap A(\epsilon)^{c}} x_{i} C_{1 \cdots n}\left(F\left(\mathbf{x}_{-n}\right), F(u)\right) f\left(\mathbf{x}_{-n}\right) \mathrm{d} \mathbf{x}_{-n}
\end{aligned}
$$

With Lemmas A.1 and A.2 we obtain

$$
\begin{aligned}
& \int_{[0, \epsilon u]^{n-1} \cap A(\epsilon)} x_{i} C_{1 \cdots n}\left(F\left(\mathbf{x}_{-n}\right), F(u(1-(n-1) \epsilon))\right) f\left(\mathbf{x}_{-n}\right) \mathrm{d} \mathbf{x}_{-n} \\
& \quad \sim \mathbb{E}\left[X_{i} 1_{\{A(\epsilon)\}} \mid X_{n}=u(1-(n-1) \epsilon)\right]+o\left(\sum_{i=1}^{n-1} \mathbb{E}\left[X_{i} \mid X_{n}=u(1-(n-1) \epsilon)\right]\right)
\end{aligned}
$$

and

$$
\begin{gathered}
\int_{[0, \epsilon u]^{n-1} \cap A(\epsilon)^{c}} x_{i} C_{1 \cdots n}\left(F\left(\mathbf{x}_{-n}\right), F(u)\right) f\left(\mathbf{x}_{-n}\right) \mathrm{d} \mathbf{x}_{-n} \\
\sim \mathbb{E}\left[X_{i} 1_{\{A(\epsilon)\}} \mid X_{n}=u\right]+o\left(\sum_{i=1}^{n-1} \mathbb{E}\left[X_{i} \mid X_{n}=u\right]\right) .
\end{gathered}
$$

It follows from Assumptions 3.2 and 3.1 that

$$
\begin{aligned}
& \int_{[0, \epsilon u]^{n-1}} x_{i} C_{1 \cdots n}\left(F\left(\mathbf{x}_{-n}\right), F\left(\xi_{\mathbf{x}_{-n}, u}\right)\right) f\left(\mathbf{x}_{-n}\right) f\left(\xi_{\mathbf{x}_{-n}, u}\right) \mathrm{d} \mathbf{x}_{-n} \\
& \lesssim\left(1+o_{\epsilon}(1)\right) f(u) \mathbb{E}\left[X_{i} \mid X_{n}=u\right]+o\left(f(u) \sum_{j \notin\{i, n\}} \mathbb{E}\left[X_{j} \mid X_{n}=u\right]\right) .
\end{aligned}
$$

A lower bound can be derived analogously, hence the lemma follows with $\epsilon \rightarrow 0$.

Proof of Theorem 3.1 Let $X_{(1)} \leq \cdots \leq X_{(n)}$ be the order statistic of $X_{1}, \ldots, X_{n}$. Following the ideas of Barbe and McCormick (2009) and Albrecher et al. (2010) we get

$$
\begin{aligned}
\mathbb{P}\left(S_{n}>u\right)= & \mathbb{P}\left(S_{n}>u, X_{(n-1)} \leq \frac{u}{2(n-1)}\right) \\
& +\mathbb{P}\left(S_{n}>u, X_{(n-1)}>\frac{u}{2(n-1)}\right)
\end{aligned}
$$




$$
\begin{aligned}
= & \sum_{i=1}^{n} \mathbb{P}\left(S_{n}>u, X_{(n-1)} \leq \frac{u}{2(n-1)}, X_{(n)}=X_{i}\right) \\
& +\sum_{i=1}^{n} \mathbb{P}\left(S_{n}>u, X_{(n-1)}>\frac{u}{2(n-1)}, X_{(n)}=X_{i}\right) .
\end{aligned}
$$

From Assumption 2.3 it follows

$$
\begin{aligned}
& \mathbb{P}\left(S_{n}>u, X_{(n-1)}>\frac{u}{2(n-1)}, X_{(n)}=X_{i}\right) \\
& \quad \leq \sum_{j=1, i \neq j}^{n} \mathbb{P}\left(X_{j}>\frac{u}{2(n-1)}, X_{i}>\frac{u}{2(n-1)}\right) \\
& \quad=\mathcal{O}\left(\sum_{j=1, i \neq j}^{n} \mathbb{P}\left(X_{j}>u, X_{i}>u\right)\right) .
\end{aligned}
$$

Next, w.l.o.g. we assume that $X_{(n)}=X_{n}$, we get

$$
\begin{aligned}
\mathbb{P} & \left(S_{n}>u, X_{(n-1)} \leq \frac{u}{2(n-1)}, X_{(n)}=X_{n}\right) \\
= & \int_{[0, \delta u]^{n-1}} \int_{u-x_{1}-\cdots-x_{n-1}}^{\infty} C_{1 \cdots n}(F(\mathbf{x})) f\left(x_{1}\right) \cdots f\left(x_{n}\right) \mathrm{d} x_{1} \cdots \mathrm{d} x_{n} \\
= & \int_{[0, u /(2(n-1))]^{n-1}} \int_{u}^{\infty} C_{1 \cdots n}(F(\mathbf{x})) f\left(x_{1}\right) \cdots f\left(x_{n}\right) \mathrm{d} x_{1} \cdots \mathrm{d} x_{n} \\
& +\int_{[0, u /(2(n-1))]^{n-1}} \int_{u-x_{1}-\cdots-x_{n-1}}^{u} C_{1 \cdots n}(F(\mathbf{x})) f\left(x_{1}\right) \cdots f\left(x_{n}\right) \mathrm{d} x_{1} \cdots \mathrm{d} x_{n} \\
= & I_{1}+I_{2} .
\end{aligned}
$$

Note that

$$
\begin{aligned}
I_{1} & =\mathbb{P}\left(X_{1} \leq \frac{u}{2(n-1)}, \cdots, X_{n-1} \leq \frac{u}{2(n-1)}, X_{n}>u\right) \\
& =\mathbb{P}\left(X_{n}>u\right)-\mathbb{P}\left(\max \left(X_{1}, \ldots, X_{n-1}\right)>\frac{u}{2(n-1)}, X_{n}>u\right) \\
& =\mathbb{P}\left(X_{n}>u\right)+\mathcal{O}\left(\sum_{i=1}^{n-1} \mathbb{P}\left(X_{i}>u, X_{n}>u\right)\right) .
\end{aligned}
$$


By the mean value theorem we get that for $u-\sum_{i=1}^{n-1} x_{i} \leq \xi_{\mathbf{x}_{-n}, u} \leq u$ and Lemmas A1-A.3

$$
\begin{aligned}
I_{2} & =\sum_{i=1}^{n-1} \int_{[0, u /(2(n-1))]^{n-1}} x_{i} C_{1 \cdots n}\left(F\left(\mathbf{x}_{-n}\right), F\left(\xi_{\mathbf{x}_{-n}, u}\right)\right) f\left(\mathbf{x}_{-n}\right) f\left(\xi_{\mathbf{x}_{-n}, u}\right) \mathrm{d} \mathbf{x}_{-n} \\
& \sim \sum_{i=1}^{n-1} \int_{[0, u /(2(n-1))]^{n-1}} x_{i} C_{1 \cdots n}\left(F\left(\mathbf{x}_{-n}\right), F(u) f\left(\mathbf{x}_{-n}\right) f(u) \mathrm{d} \mathbf{x}_{-n}\right. \\
& \sim \sum_{i=1}^{n-1} \mathbb{E}\left[X_{i} \mid X_{n}=u\right],
\end{aligned}
$$

hence the proof is complete.

Proof of Corollary 3.2 This follows from Theorem 3.1, since for a function $a(u)$ with $\lim _{u \rightarrow \infty} a(u) / u=0$

$$
\mathbb{P}\left(X_{i}>u-a(u)\right)=\mathbb{P}\left(X_{i}>u\right)+a(u) f\left(u-\xi_{u}\right) \sim \mathbb{P}\left(X_{i}>u\right)+a(u) f(u),
$$

where $0<\xi(u)<a(u)$.

\section{Appendix B: Proofs for the Gaussian copula}

At first note that for $x \rightarrow \infty$ and $z \rightarrow 0$

$$
1-\Phi(x) \sim \frac{1}{x \sqrt{2 \pi}} e^{-\frac{x^{2}}{2}}=\frac{1}{x} \phi(x) \text { and } \Phi^{-1}(1-z) \sim \sqrt{-2 \log (z)} .
$$

Further note that

$$
\lim _{z \rightarrow 0} \Phi^{-1}(1-z)\left(\Phi^{-1}(1-z)-\left(\sqrt{-2 \log (z)}-\frac{\log (-\log (z))}{2 \sqrt{-2 \log (z)}}\right)\right)=\frac{1}{2} \log (4 \pi) .
$$

Throughout the proofs we denote with $\bar{a}=\Phi^{-1}(F(a))$ and with $Y_{1}, \ldots, Y_{n} n$ i.i.d. standard normal random variables. Before we prove Proposition 4.1, we prove Propositions 4.2 and 4.3

Proof of Proposition 4.2 Denoting with $Y_{1}$ and $Y_{2}$ two standard normal random variables, we have

$$
\begin{aligned}
\mathbb{E}\left[X_{2} \mid X_{1}=u\right] & =\mathbb{E}\left[F^{-1}\left(\Phi\left(\rho Y_{1}+\sqrt{1-\rho^{2}} Y_{2}\right)\right) \mid F^{-1}\left(\Phi\left(Y_{1}\right)\right)=u\right] \\
& =\mathbb{E}\left[F^{-1}\left(\Phi\left(\rho \bar{u}+\sqrt{1-\rho^{2}} Y_{2}\right)\right)\right]
\end{aligned}
$$




$$
\begin{aligned}
& =\mathbb{E}\left[F^{-1}\left(\Phi\left(\rho \bar{u}+\sqrt{1-\rho^{2}} Y_{2}\right)\right) 1_{\left\{Y_{2}>0\right\}}\right] \\
& +\mathbb{E}\left[F^{-1}\left(\Phi\left(\rho \bar{u}+\sqrt{1-\rho^{2}} Y_{2}\right)\right) 1_{\left\{Y_{2} \leq 0\right\}}\right] .
\end{aligned}
$$

Note that with Potter bounds (Bingham et al. 1989)

$\mathbb{E}\left[F^{-1}\left(\Phi\left(\rho \bar{u}+\sqrt{1-\rho^{2}} Y_{2}\right)\right) 1_{\left\{Y_{2} \leq 0\right\}}\right] \leq F^{-1}\left(\Phi\left(\rho \Phi^{-1}(F(u))\right)\right) \lesssim K u^{\rho^{2}+\delta}$,

for every $\delta>0$ and $K>1$. We get for every $\delta>0$ and $\epsilon>0$ that uniformly for $\sqrt{1-\rho^{2}} Y_{2} / \bar{u}=x \geq-\rho+\delta$

$$
\begin{aligned}
& F^{-1}(\Phi((\rho+x) \bar{u})) \sim F^{-1}\left(1-\frac{\exp \left(-\frac{1}{2}((\rho+x) \bar{u})^{2}\right)}{\sqrt{2 \pi}(\rho+x) \bar{u}}\right) \\
& =F^{-1}\left(1-\left(\frac{e^{-\frac{\Phi^{-1}(F(u))^{2}}{2}}}{\sqrt{2 \pi} \Phi^{-1}(F(u))}\right)^{(x+\rho)^{2}} \frac{(\sqrt{2 \pi} \bar{u})^{(x+\rho)^{2}-1}}{(\rho+x)}\right) \\
& \lesssim F^{-1}\left(1-\frac{((1-\epsilon) \sqrt{2 \pi} \bar{u} \bar{F}(u))^{(x+\rho)^{2}}}{(\rho+x) \sqrt{2 \pi} \bar{u}}\right) \\
& =\kappa\left(\frac{((1-\epsilon) \sqrt{2 \pi} \bar{u} \bar{F}(u))^{(x+\rho)^{2}}}{(\rho+x) \sqrt{2 \pi} \bar{u}}\right)^{-1 / \alpha} \\
& +r\left(\frac{((1-\epsilon) \sqrt{2 \pi} \bar{u} \bar{F}(u))^{(x+\rho)^{2}}}{(\rho+x) \sqrt{2 \pi} \bar{u}}\right) .
\end{aligned}
$$

Further

$$
r\left(\frac{((1-\epsilon) \sqrt{2 \pi} \bar{u} \bar{F}(u))^{(x+\rho)^{2}}}{(\rho+x) \sqrt{2 \pi} \bar{u}}\right) \leq K\left(\frac{((1-\epsilon) \sqrt{2 \pi} \bar{u} \bar{F}(u))^{(x+\rho)^{2}}}{(\rho+x) \sqrt{2 \pi} \bar{u}}\right)^{-\beta} .
$$


For $g(u):=(1-\epsilon) \sqrt{2 \pi} \bar{u} \bar{F}(u)$,

$$
\lim _{u \rightarrow \infty} \frac{-\log (g(u))}{\bar{u}^{2}}=\frac{1}{2} .
$$

Next we evaluate the asymptotics of

$$
\begin{aligned}
\mathbb{E}\left[F^{-1}\left(\Phi\left(\rho \bar{u}+\sqrt{1-\rho^{2}} Y_{2}\right)\right) 1_{\left\{Y_{2}>0\right\}}\right] \\
\lesssim \frac{\kappa}{\sqrt{2 \pi}} \int_{0}^{\infty}\left(\frac{(g(u))^{\left(\sqrt{1-\rho^{2}} \frac{x}{\bar{u}}+\rho\right)^{2}}}{\left(\rho+\sqrt{1-\rho^{2}} \frac{x}{\bar{u}}\right) \sqrt{2 \pi} \bar{u}}\right)^{-1 / \alpha} e^{-\frac{x^{2}}{2}} \mathrm{~d} x \\
=\frac{\kappa(\sqrt{2 \pi} \bar{u})^{1 / \alpha}}{\sqrt{2 \pi}} \int_{0}^{\infty}\left(\rho+\sqrt{1-\rho^{2}} \frac{x}{\bar{u}}\right)^{1 / \alpha} \\
\quad \times \exp \left(\frac{(-2 \log (g(u)))\left(\rho+\sqrt{1-\rho^{2}} \frac{x}{\bar{u}}\right)^{2}-\alpha x^{2}}{2 \alpha}\right) \mathrm{d} x .
\end{aligned}
$$

The exponent is maximized for

$$
x_{u}=\frac{-2 \log (g(u)) \rho \sqrt{1-\rho^{2}}}{\bar{u}}\left(\alpha-\frac{-2 \log (g(u))\left(1-\rho^{2}\right)}{\bar{u}^{2}}\right)^{-1} \sim \bar{u} \frac{\rho \sqrt{1-\rho^{2}}}{\alpha+\rho^{2}-1} .
$$

Substitution $x-x_{u}=y$ yields

$$
\begin{aligned}
& \mathbb{E}\left[F ^ { - 1 } \left(\Phi \left(\rho \bar{u}+\sqrt{\left.\left.\left.1-\rho^{2} Y_{2}\right)\right) 1_{\left\{Y_{2}>0\right\}}\right]}\right.\right.\right. \\
& \quad \sim \frac{\kappa(\sqrt{2 \pi} \bar{u})^{1 / \alpha}}{\sqrt{2 \pi}} \exp \left(\frac{(-\log (g(u))) \rho^{2}}{\alpha+\frac{-2 \log (g(u))}{\bar{u}^{2}}\left(\rho^{2}-1\right)}\right) \\
& \quad \times \int_{-x_{u}}^{\infty}\left(\rho+\sqrt{1-\rho^{2}} \frac{x+x_{u}}{\bar{u}}\right)^{1 / \alpha} \exp \left(\frac{\left(\frac{(-2 \log (g(u)))\left(1-\rho^{2}\right)}{\bar{u}^{2}}-\alpha\right) x^{2}}{2 \alpha}\right) \mathrm{d} x \\
& \quad \kappa(\sqrt{2 \pi} \bar{u})^{1 / \alpha}\left(\frac{\rho \alpha}{\alpha+\rho^{2}-1}\right)^{1 / \alpha} \sqrt{\frac{\alpha}{\alpha+\rho^{2}-1}} \\
& \quad \times \exp \left(\frac{(-\log (g(u))) \rho^{2}}{\alpha+\frac{-2 \log (g(u))}{\bar{u}^{2}}\left(\rho^{2}-1\right)}\right) .
\end{aligned}
$$


To finish the proof, note that

$$
\begin{aligned}
& \exp \left(\frac{\left(-\log (g(u)) \rho^{2}\right.}{\alpha+\frac{-2 \log (g(u))}{\bar{u}^{2}}\left(\rho^{2}-1\right)}\right) \\
& \lesssim \exp \left(\frac{\left(-\log (g(u)) \rho^{2}\right.}{\alpha+\rho^{2}-1}+\frac{(-\log (g(u))}{\bar{u}^{2}}\right. \\
& \left.\quad \times\left(-2 \log (g(u))-\bar{u}^{2}\right) \frac{\rho^{2}\left(1-\rho^{2}\right)}{\left(\alpha+\xi_{u}\left(\rho^{2}-1\right)\right)^{2}}\right) .
\end{aligned}
$$

We have

$$
\begin{aligned}
& -2 \log (g(u))-\bar{u}^{2} \\
& \sim-2 \log (1-\epsilon)-\log (2 \pi)-2 \log (\bar{F}(u))-\log \left(\Phi^{-1}(F(u))^{2}\right) \\
& -\left(\sqrt{-2 \log (\bar{F}(u))}-\frac{\log (-\log (\bar{F}(u)))}{2 \sqrt{-2 \log (\bar{F}(u))}}\right)^{2}+\log (4 \pi) \\
& =-2 \log (1-\epsilon)+\log (2)+\log \left(\frac{-\log (\bar{F}(u))}{\Phi^{-1}(F(u))^{2}}\right)-\left(\frac{\log (-\log (\bar{F}(u)))}{2 \sqrt{-2 \log (\bar{F}(u))}}\right)^{2} \\
& \rightarrow-2 \log (1-\epsilon) .
\end{aligned}
$$

Hence for $k(\epsilon):=(1-\epsilon)^{-\frac{\rho^{2}\left(1-\rho^{2}\right)}{\left(\alpha+\left(\rho^{2}-1\right)\right)^{2}}}$

$$
\begin{aligned}
& \mathbb{E}\left[X_{2} \mid X_{1}=u\right] \\
& \lesssim \kappa k(\epsilon)(\sqrt{2 \pi} \bar{u})^{1 / \alpha}\left(\frac{\rho \alpha}{\alpha+\rho^{2}-1}\right)^{1 / \alpha} \sqrt{\frac{\alpha}{\alpha+\rho^{2}-1}} \exp \left(\frac{\left(-\log (g(u)) \rho^{2}\right.}{\alpha+\left(\rho^{2}-1\right)}\right) \\
& =\kappa k(\epsilon)\left(\frac{\rho \alpha}{\alpha+\rho^{2}-1}\right)^{1 / \alpha} \sqrt{\frac{\alpha}{\alpha+\rho^{2}-1}}(\sqrt{2 \pi} \bar{u})^{\frac{1}{\alpha}-\frac{\rho^{2}}{\alpha+\rho^{2}-1}} \bar{F}(u)^{-\frac{\rho^{2}}{\alpha+\rho^{2}-1}} \\
& \sim \kappa k(\epsilon)\left(\frac{\rho \alpha}{\alpha+\rho^{2}-1}\right)^{1 / \alpha} \sqrt{\frac{\alpha}{\alpha+\rho^{2}-1}} \\
& \quad \times(-4 \pi \log (\bar{F}(u)))^{\frac{1}{2 \alpha}-\frac{\rho^{2}}{2\left(\alpha+\rho^{2}-1\right)} \bar{F}(u)^{-\frac{\rho^{2}}{\alpha+\rho^{2}-1}} .}
\end{aligned}
$$

An asymptotic lower bound can be established analogously. The propositions follows with $\epsilon \rightarrow 0$. 
Proof of Proposition 4.3 For $\delta>0$ we have to investigate the following three cases

$$
\begin{aligned}
\mathbb{E}\left[X_{2} \mid X_{1}=u\right]= & \mathbb{E}\left[F^{-1}\left(\Phi\left(\rho \bar{u}+\sqrt{1-\rho^{2}} Y_{2}\right)\right) 1_{\left.\left\{\frac{\sqrt{1-\rho^{2}} Y_{2}}{\bar{u}}<-\rho-\delta\right\}\right]}\right. \\
+ & \mathbb{E}\left[F^{-1}\left(\Phi\left(\rho \bar{u}+\sqrt{1-\rho^{2}} Y_{2}\right)\right) 1_{\left.\left\{-\rho-\delta<\frac{\sqrt{1-\rho^{2}} Y_{2}}{\bar{u}}<-\rho+\delta\right\}\right]}\right] \\
+ & \mathbb{E}\left[F^{-1}\left(\Phi\left(\rho \bar{u}+\sqrt{1-\rho^{2}} Y_{2}\right)\right) 1_{\left.\left\{\frac{\sqrt{1-\rho^{2}} Y_{2}}{\bar{u}}>-\rho+\delta\right\}\right]}\right.
\end{aligned}
$$

At first we consider Eq. 16. By the same method as in the proof of Proposition 4.2, we get by Potter bounds that for each $K>1$ and $\epsilon>0$

$$
\begin{aligned}
& \mathbb{E}\left[F^{-1}\left(\Phi\left(\rho \bar{u}+\sqrt{1-\rho^{2}} Y_{2}\right)\right) 1_{\left.\left\{\frac{\sqrt{1-\rho^{2}} Y_{2}}{\bar{u}}>-\rho+\delta\right\}\right]}\right. \\
& \left.\lesssim K \frac{(\sqrt{2 \pi} \bar{u})^{\frac{1}{\alpha-\epsilon}}}{\sqrt{2 \pi}} \int_{\frac{-\rho+\delta}{\sqrt{1-\rho^{2}}} \bar{u}}^{\infty}\left(\rho+\sqrt{1-\rho^{2}} \frac{x}{\bar{u}}\right)^{\frac{1}{\alpha-\epsilon}}\right) \\
& \quad \times \exp \left(\frac{(-2 \log (g(u)))\left(\rho+\sqrt{1-\rho^{2}} \frac{x}{\bar{u}}\right)^{2}-(\alpha-\epsilon) x^{2}}{2(\alpha-\epsilon)}\right)
\end{aligned}
$$

The exponent is maximized for

$$
\begin{aligned}
x_{u} & =\frac{-2 \log (g(u)) \rho \sqrt{1-\rho^{2}}}{\bar{u}}\left((\alpha-\epsilon)-\frac{-2 \log (g(u))\left(1-\rho^{2}\right)}{\bar{u}^{2}}\right)^{-1} \\
& \sim \bar{u} \frac{\rho \sqrt{1-\rho^{2}}}{\alpha-\epsilon+\rho^{2}-1}<0 .
\end{aligned}
$$

Since $\alpha>1-\rho^{2}$, we get that the derivative of the exponent at the point $\hat{x}_{u}=$ $-\rho \bar{u} / \sqrt{1-\rho^{2}}$ is negative. Hence we can bound

$$
\begin{aligned}
& \exp \left(\frac{(-2 \log (g(u)))\left(\rho+\sqrt{1-\rho^{2}} \frac{x}{\bar{u}}\right)^{2}-(\alpha-\epsilon) x^{2}}{2(\alpha-\epsilon)}\right) \\
& \leq e^{\frac{-\rho^{2} \bar{u}^{2}}{2\left(1-\rho^{2}\right)}} \exp \left(\frac{\frac{-2 \log (g(u))}{\bar{u}^{2}}\left(1-\rho^{2}\right)-(\alpha-\epsilon)}{2(\alpha-\epsilon)}\left(x-\hat{x}_{u}\right)^{2}\right) .
\end{aligned}
$$


It follows that for $K_{1}>1$ and $\epsilon_{1}>0$ we can choose $\delta$ such that

$$
\mathbb{E}\left[F^{-1}\left(\Phi\left(\rho \bar{u}+\sqrt{1-\rho^{2}} Y_{2}\right)\right) 1_{\left\{\frac{\sqrt{1-\rho^{2}} Y_{2}}{\bar{u}}>-\rho+\delta\right\}}\right] \lesssim K_{1} \bar{F}(u)^{\frac{\rho^{2}-\epsilon_{1}}{1-\rho^{2}}}
$$

For Eq. 15 note that for $K_{1}>1$ and $\epsilon_{1}>0$ we can choose $\delta$ such that

$$
\begin{gathered}
\mathbb{E}\left[F^{-1}\left(\Phi\left(\rho \bar{u}+\sqrt{1-\rho^{2}} Y_{2}\right)\right) 1\left\{-\rho-\delta<\frac{\sqrt{1-\rho^{2}} Y_{2}}{\bar{u}}<-\rho+\delta\right\}\right] \\
\leq \bar{F}^{-1}(\Phi(\delta \bar{u})) \mathbb{P}\left(Y_{2}>\frac{-(\rho+\delta)}{\sqrt{1-\rho^{2}}} \bar{u}\right) \lesssim K_{1} \bar{F}(u)^{\frac{\rho^{2}-\epsilon_{1}}{1-\rho^{2}}} .
\end{gathered}
$$

We are left with finding the asymptotic of Eq. 14. If $x_{F}>0$, the Proposition follows since uniformly for $u \rightarrow \infty(\delta<-\rho)$

$$
\left(\rho \bar{u}+\sqrt{1-\rho^{2}} Y_{2}\right) 1_{\left\{\frac{\sqrt{1-\rho^{2}} Y_{2}}{\bar{u}}<-\rho-\delta\right\}} \rightarrow-\infty \quad \text { and } \quad 1_{\left\{\frac{\sqrt{1-\rho^{2}} Y_{2}}{\bar{u}}<-\rho-\delta\right\}} \rightarrow 1 .
$$

If $x_{F}=0$ we get, analogous to the proof of Proposition 4.2 for $x<-(\rho+\delta)$

$$
\begin{aligned}
& F^{-1}(\Phi((\rho+x) \bar{u})) \sim F^{-1}\left(\frac{\exp \left(-\frac{1}{2}((\rho+x) \bar{u})^{2}\right)}{-\sqrt{2 \pi}(\rho+x) \bar{u}}\right) \\
& \lesssim F^{-1}\left(\frac{\left.((1+\epsilon) \sqrt{2 \pi} \bar{u} \bar{F}(u))^{(x+\rho)^{2}}\right)}{-(\rho+x) \sqrt{2 \pi} \bar{u}}\right) \\
&=\kappa\left(\frac{\left.((1+\epsilon) \sqrt{2 \pi} \bar{u} \bar{F}(u))^{(x+\rho)^{2}}\right)^{\tau}}{-(\rho+x) \sqrt{2 \pi} \bar{u}}\right)^{(x+\rho)^{2}} \\
&+r\left(\frac{((1+\epsilon) \sqrt{2 \pi} \bar{u} \bar{F}(u))^{(}}{-(\rho+x) \sqrt{2 \pi} \bar{u}}\right) .
\end{aligned}
$$

Further we have that

$$
r_{1}\left(\frac{((1+\epsilon) \sqrt{2 \pi} \bar{u} \bar{F}(u))^{(x+\rho)^{2}}}{-(\rho+x) \sqrt{2 \pi} \bar{u}}\right) \leq K_{1}\left(\frac{((1+\epsilon) \sqrt{2 \pi} \bar{u} \bar{F}(u))^{(x+\rho)^{2}}}{-(\rho+x) \sqrt{2 \pi} \bar{u}}\right)^{\beta_{1}}
$$


For $g(u):=(1+\epsilon) \sqrt{2 \pi} \bar{u} \bar{F}(u)$ we have that

$$
\begin{aligned}
\mathbb{E}\left[F^{-1}\left(\Phi\left(\rho \bar{u}+\sqrt{1-\rho^{2}} Y_{2}\right)\right) 1\left\{\frac{\sqrt{1-\rho^{2}} Y_{2}}{\bar{u}}<-\rho-\delta\right\}\right] \\
\lesssim \frac{\kappa(\sqrt{2 \pi} \bar{u})^{-\tau}}{\sqrt{2 \pi}} \int_{-\infty}^{\frac{-(\rho+\delta)}{\sqrt{1-\rho^{2}}}\left(-\rho-\sqrt{1-\rho^{2}} \frac{x}{\bar{u}}\right)^{-\tau}} \\
\quad \times \exp \left(-\frac{\tau(-2 \log (g(u)))\left(\rho+\sqrt{1-\rho^{2}} \frac{x}{\bar{u}}\right)^{2}+x^{2}}{2}\right) \mathrm{d} x .
\end{aligned}
$$

The exponent is maximized for

$$
\begin{aligned}
x_{u} & =-\frac{-2 \log (g(u)) \tau \rho \sqrt{1-\rho^{2}}}{\bar{u}}\left(1+\frac{-2 \log (g(u)) \tau\left(1-\rho^{2}\right)}{\bar{u}^{2}}\right)^{-1} \\
& \sim-\bar{u} \frac{\tau \rho \sqrt{1-\rho^{2}}}{1+\tau\left(1-\rho^{2}\right)} .
\end{aligned}
$$

Continuing as in the proof of Proposition 4.2 we get that there exists $k(\epsilon) \rightarrow 1$ as $\epsilon \rightarrow 0$ with

$$
\begin{aligned}
\mathbb{E}\left[F^{-1}\left(\Phi\left(\rho \bar{u}+\sqrt{1-\rho^{2}} Y_{2}\right)\right) 1_{\left.\left\{\frac{\sqrt{1-\rho^{2}} Y_{2}}{\bar{u}}<-\rho-\delta\right\}\right]}\right)^{-\tau} \sqrt{\frac{1}{1+\tau\left(1-\rho^{2}\right)}} \\
\lesssim \kappa k(\epsilon)(\sqrt{2 \pi} \bar{u})^{-\tau}\left(\frac{-\rho}{1+\tau\left(1-\rho^{2}\right)}\right) \\
\quad \times \exp \left(-\frac{(-\log (g(u))) \tau \rho^{2}}{1+\frac{-2 \log (g(u))}{\bar{u}^{2}} \tau\left(1-\rho^{2}\right)}\right) \\
\sim \kappa k(\epsilon)\left(\frac{-\rho}{1+\tau\left(1-\rho^{2}\right)}\right)^{-\tau} \sqrt{\frac{1+\tau\left(1-\rho^{2}\right)}{1+\rho^{2}}} \\
\quad \times(-4 \pi \log (\bar{F}(u)))^{-\frac{\tau}{2}+\frac{\tau \rho^{2}}{2\left(1+\tau\left(1-\rho^{2}\right)\right)} \bar{F}(u)^{\frac{\tau \rho^{2}}{1+\tau\left(1-\rho^{2}\right)}} .}
\end{aligned}
$$

As in the proof of Proposition 4.2 we can get a similar lower bound. To finish the proof note that

$$
\frac{\tau \rho^{2}}{1+\tau\left(1-\rho^{2}\right)}<\frac{\rho^{2}}{1-\rho^{2}} .
$$


Proof of Proposition 4.1 To prove Assumptions 2.1-2.3 we can w.l.o.g. assume that $n=2$ and $\rho:=\rho_{i, j}$. From Ledford and Tawn (1996, Eq. 5.1) it follows that

$$
\begin{aligned}
\mathbb{P}\left(X_{1}>u, X_{2}>u\right) & \sim C_{\rho}\left(-\log (F(u))^{\frac{2}{1+\rho}}(-\log (-\log (F(u))))^{-\frac{\rho}{1+\rho}}\right. \\
& \sim C_{\rho} \bar{F}(u)^{\frac{2}{1+\rho}}(-\log (\bar{F}(u)))^{-\frac{\rho}{1+\rho}}
\end{aligned}
$$

where $C_{\rho}=(1+\rho)^{3 / 2}(1-\rho)^{-1 / 2}(4 \pi)^{-\rho /(1+\rho)}$. Hence Assumption 2.3 holds.

For Assumptions 2.1 and 2.2 note that

$$
\begin{aligned}
& \int_{1-\delta a}^{1} C_{1,2}(x, 1-y a) \mathrm{d} x \\
& =\int_{1-\delta a}^{1} \frac{\frac{1}{2 \pi \sqrt{1-\rho^{2}}} \exp \left(-\frac{\Phi^{-1}(x)^{2}+\Phi^{-1}(1-y a)^{2}-2 \rho \Phi^{-1}(x) \Phi^{-1}(1-y a)}{2\left(1-\rho^{2}\right)}\right)}{\phi\left(\Phi^{-1}(x)\right) \phi\left(\Phi^{-1}(1-y a)\right)} \mathrm{d} x \\
& =1-\Phi\left(\frac{\Phi^{-1}(1-\delta a)-\rho \Phi^{-1}(1-y a)}{\sqrt{1-\rho^{2}}}\right)
\end{aligned}
$$

To prove Assumption 2.1 note that for all $0<\epsilon_{1}<1, \epsilon_{2}>0$ and uniformly for $y \in\left(\epsilon_{1}, 1+\epsilon_{2}\right) \Phi^{-1}(1-y a) \sim \Phi^{-1}(1-a)$. Hence for constant $d_{1}>0$ there exists $0<a_{0}<1$ such that for $y \in\left(\epsilon_{1}, 1+\epsilon_{2}\right)$ and $a_{0}<a<1$

$$
\begin{aligned}
& \frac{1-\Phi\left(\frac{\Phi^{-1}(1-a)-\rho \Phi^{-1}(1-y a)}{\sqrt{1-\rho^{2}}}\right)}{1-\Phi\left(\frac{\Phi^{-1}(1-a)-\rho \Phi^{-1}(1-a)}{\sqrt{1-\rho^{2}}}\right)} \\
& \leq d_{1} \exp \left(-\frac{\rho^{2}\left(\Phi^{-1}(1-y a)^{2}-\Phi^{-1}(1-a)^{2}\right)-2 \rho \Phi^{-1}(1-a)\left(\Phi^{-1}(1-y a)-\Phi^{-1}(1-a)\right)}{2\left(1-\rho^{2}\right)}\right) .
\end{aligned}
$$

With Eq. 13 we get that

$$
\begin{aligned}
\Phi^{-1}(1-y a)^{2}-\Phi^{-1}(1-a)^{2} \\
=\left(\sqrt{-2 \log (y a)}-\frac{\log (-\log (y a))}{2 \sqrt{-2 \log (y a)}}\right)^{2} \\
\quad-\left(\sqrt{-2 \log (a)}-\frac{\log (-\log (a))}{2 \sqrt{-2 \log (a)}}\right)^{2}+o_{a}(1) \\
=-2 \log (y)-\log \left(\frac{\log (y a)}{\log (a)}\right)+o_{a}(1)=-2 \log (y)+o_{a}(1) .
\end{aligned}
$$


Analogously

$$
\begin{aligned}
& \Phi^{-1}(1-a)\left(\Phi^{-1}(1-y a)-\Phi^{-1}(1-a)\right) \\
& =\Phi^{-1}(1-a)\left(\sqrt{-2 \log (y a)}-\sqrt{-2 \log (a)}+\frac{\log (-\log (a))}{2 \sqrt{-2 \log (a)}}\right. \\
& \left.\quad-\frac{\log (-\log (y a))}{2 \sqrt{-2 \log (y a)}}\right)+o_{a}(1) \\
& =-\log (y)+o_{a}(1) .
\end{aligned}
$$

Consequently, for $d_{2}>d_{1}$

$$
\frac{1-\Phi\left(\frac{\Phi^{-1}(1-a)-\rho \Phi^{-1}(1-y a)}{\sqrt{1-\rho^{2}}}\right)}{1-\Phi\left(\frac{\Phi^{-1}(1-a)-\rho \Phi^{-1}(1-a)}{\sqrt{1-\rho^{2}}}\right)} \leq d_{2} y^{-\frac{\rho}{1+\rho}}
$$

For Assumption 2.2 note that we get for any $d_{3}>1$, uniformly for $\delta<y<M$

$$
\begin{aligned}
& \frac{1-\Phi\left(\frac{\Phi^{-1}(1-y a)-\rho \Phi^{-1}(1-a)}{\sqrt{1-\rho^{2}}}\right)}{1-\Phi\left(\frac{\Phi^{-1}(1-\delta a)-\rho \Phi^{-1}(1-a)}{\sqrt{1-\rho^{2}}}\right)} \\
& \leq d_{3} \exp \left(-\frac{\left(\Phi^{-1}(1-y a)^{2}-\Phi^{-1}(1-\delta a)^{2}\right)-2 \rho \Phi^{-1}(1-a)\left(\Phi^{-1}(1-y a)-\Phi^{-1}(1-\delta a)\right)}{2\left(1-\rho^{2}\right)}\right) \\
& \lesssim d_{4}(y / \delta)^{\frac{1}{1+\rho}}
\end{aligned}
$$

for all $d_{4}>d_{3}$. A lower bound follows analogously. Further for $\delta=\left(1+\epsilon_{0}\right)(2(n-$ $1))^{\alpha}$ and uniformly in $y \in(0, \delta]$

$$
\begin{aligned}
\frac{1-}{-} & \Phi\left(\frac{\Phi^{-1}(1-y a)-\rho \Phi^{-1}(1-a)}{\sqrt{1-\rho^{2}}}\right) \\
1- & \Phi\left(\frac{\Phi^{-1}(1-\delta a)-\rho \Phi^{-1}(1-a)}{\sqrt{1-\rho^{2}}}\right) \\
\sim & \frac{\Phi^{-1}(1-y a)-\rho \Phi^{-1}(1-a)}{\Phi^{-1}(1-\delta a)-\rho \Phi^{-1}(1-a)} \\
& \quad \times \exp \left(-\frac{\left(\Phi^{-1}(1-y a)^{2}-\Phi^{-1}(1-\delta a)^{2}\right)-2 \rho \Phi^{-1}(1-a)\left(\Phi^{-1}(1-y a)-\Phi^{-1}(1-\delta a)\right)}{2\left(1-\rho^{2}\right)}\right)
\end{aligned}
$$

Not that uniformly for $y \in(0, \delta]$

$$
\frac{\Phi^{-1}(1-y a)-\rho \Phi^{-1}(1-a)}{\Phi^{-1}(1-\delta a)-\rho \Phi^{-1}(1-a)} \sim \frac{\sqrt{-2 \log (y a)}-\rho \sqrt{-2 \log (a)}}{(1-\rho) \sqrt{-2 \log (a)}} \lesssim d_{5} y^{-\epsilon}
$$


for all $\epsilon>0$ and $d_{5}>1$. As in Eq. 17 we get that for all $\epsilon>0$ and uniformly for $y \in(0, \delta]$

$$
\begin{aligned}
& \Phi^{-1}(1-y a)^{2}-\Phi^{-1}(1-\delta a)^{2} \sim-2 \log (y / \delta)+\log \left(\frac{\log (y a)}{\log (\delta a)}\right)+o_{a}(1) \\
& \geq-2(1-\epsilon) \log (y / \delta)+o_{a}(1)
\end{aligned}
$$

and analogously to Eq. 18 we get that for all $\epsilon>0$ and uniformly for $y \in(0, \delta]$

$$
\Phi^{-1}(1-a)\left[\Phi^{-1}(1-y a)-\Phi^{-1}(1-\delta a)\right] \leq-(1+\epsilon) \log (1+y)+o_{a}(1) .
$$

It follows that for every $\epsilon>0$, there exists a $d_{5}>0$ such that uniformly for $y \in(0, \delta]$

$$
\frac{1-\Phi\left(\frac{\Phi^{-1}(1-y a)-\rho \Phi^{-1}(1-a)}{\sqrt{1-\rho^{2}}}\right)}{1-\Phi\left(\frac{\Phi^{-1}(1-\delta a)-\rho \Phi^{-1}(1-a)}{\sqrt{1-\rho^{2}}}\right)} \lesssim d_{5} y^{-\frac{1+\epsilon}{1+\rho}}
$$

Hence Assumption 2.2 holds for $\alpha>1+\rho$.

The validity of Assumption 3.1 can be seen from the proofs of Propositions 4.2 and 4.3.

For Assumption 3.2, note that for $\Sigma^{-1}=:\left(\sigma_{i, j}^{-1}\right)_{i=1, \ldots, n, j=1, \ldots, n}$

$$
\begin{aligned}
& \frac{C_{1 \cdots n}\left(F\left(y_{1}\right), \ldots, F\left(y_{n-1}\right), F(u(1-x))\right.}{C_{1 \cdots n}\left(F\left(y_{1}\right), \ldots, F\left(y_{n-1}\right), F(u)\right.} \\
& =\frac{\phi\left(\Phi^{-1}(F(u))\right)}{\phi\left(\Phi^{-1}(F(u(1-x)))\right)} \exp \left(-\frac{\sigma_{n, n}^{-1}}{2}\left(\Phi^{-1}(F(u(1-x)))^{2}-\Phi^{-1}(F(u))^{2}\right)\right) \\
& \quad \times \exp \left(-\sum_{i=1}^{n-1} \sigma_{i, n}^{-1} \Phi^{-1}\left(F\left(y_{i}\right)\right)\left(\Phi^{-1}(F(u(1-x)))-\Phi^{-1}(F(u))\right)\right) .
\end{aligned}
$$

We get uniformly for $0<x<1 / 2$

$$
\begin{aligned}
& \frac{\phi\left(\Phi^{-1}(F(u))\right)}{\phi\left(\Phi^{-1}(F(u(1-x)))\right)} \exp \left(-\frac{\sigma_{n, n}^{-1}}{2}\left(\Phi^{-1}(F(u(1-x)))^{2}-\Phi^{-1}(F(u))^{2}\right)\right) \\
& =\left(\frac{\phi\left(\Phi^{-1}(F(u))\right)}{\phi\left(\Phi^{-1}(F(u(1-x)))\right)}\right)^{1-\sigma_{n, n}^{-1}} \\
& \sim\left(\frac{\sqrt{-2 \log \bar{F}(u)} \bar{F}(u)}{\sqrt{-2 \log \bar{F}(u(1-x))} \bar{F}(u(1-x))}\right)^{1-\sigma_{n, n}^{-1}} \\
& \sim(1-x)^{\alpha\left(1-\sigma_{n, n}^{-1}\right)} .
\end{aligned}
$$


Further we get uniformly for $\epsilon<F\left(y_{i}\right)<F(u /(2(n-1)))$ as above

$$
\begin{aligned}
& \exp \left(-\sum_{i=1}^{n-1} \sigma_{i, n}^{-1} \Phi^{-1}\left(F\left(y_{i}\right)\right)\left(\Phi^{-1}(F(u(1-x)))-\Phi^{-1}(F(u))\right)\right) \\
& \sim \exp \left(-\alpha \sum_{i=1}^{n-1} \sigma_{i, n}^{-1} \frac{\Phi^{-1}\left(F\left(y_{i}\right)\right)}{\Phi^{-1}(F(u))} \log (1-x)\right) .
\end{aligned}
$$

Since $\frac{\Phi^{-1}\left(F\left(y_{i}\right)\right)}{\Phi^{-1}(F(u))} \leq 1$, we get that for

$$
A(\epsilon)=\left\{\sum_{i=1}^{n-1} \sigma_{i, n}^{-1} \Phi^{-1}\left(F\left(y_{i}\right)\right)<0\right\} \backslash\left\{F\left(y_{i}\right)>\epsilon, i=1, \ldots, n-1\right\},
$$

the bounds where $F(u(1-x))$ is replaced by $F(u)$ in Assumption 3.2 are fulfilled. The bounds where $F(u(1-x))$ is replaced by $F(u(1-(n-1) \epsilon))$ follow analogously. To prove that $A(\epsilon)$ fulfills Eq. 6 we have to show that

$$
\mathbb{E}\left[X_{i} 1_{\{A(\epsilon)\}} \mid X_{n}=u\right] \sim\left(1+o_{\epsilon}(1)\right) \mathbb{E}\left[X_{i} 1_{\{A(\epsilon)\}} \mid X_{n}=u(1-(n-1) \epsilon)\right] .
$$

or

$$
\begin{aligned}
\mathbb{E}\left[X_{i} 1_{\{A(\epsilon)\}} \mid X_{n}=u\right] & =o\left(\mathbb{E}\left[X_{i} \mid X_{n}=u\right]\right) \quad \text { and } \\
\mathbb{E}\left[X_{i} 1_{\{A(\epsilon)\}} \mid X_{n}=u(1-(n-1) \epsilon)\right] & =o\left(\mathbb{E}\left[X_{i} \mid X_{n}=u\right]\right)
\end{aligned}
$$

W.l.o.g. we can choose $i=1$ and for i.i.d. standard normal $Y_{1}, \ldots, Y_{n}$ (Cholesky decomposition)

$X_{n}=F^{-1}\left(\Phi\left(Y_{n}\right)\right), \quad X_{i}=F^{-1}\left(\Phi\left(\rho_{i, n} Y_{n}+\sum_{j=1}^{i} \hat{\rho}_{i, j} Y_{j}\right)\right), \quad i=1, \ldots, n-1$

where $\hat{\rho}_{1,1}=\sqrt{1-\rho_{1, n}^{2}}$. Define

$$
X_{i}(u)=\left(X_{i} \mid X_{n}=u\right):=\bar{F}^{-1}\left(\Phi\left(\rho_{i, n} \bar{u}+\sum_{j=1}^{i} \hat{\rho}_{i, j} Y_{j}\right)\right)
$$

and

$$
A(\epsilon, u)=\left\{\sum_{i=1}^{n-1} \sigma_{i, n}^{-1} \Phi^{-1}\left(F\left(X_{i}(u)\right)\right)<0\right\} \backslash\left\{F\left(X_{i}(u)\right)>\epsilon, i=1, \ldots, n-1\right\} .
$$

Then

$$
\begin{aligned}
\mathbb{E}\left[X_{i} 1_{\{A(\epsilon)\}} \mid X_{n}=u\right] & =\mathbb{E}\left[X_{i}(u) 1_{\{A(\epsilon, u)\}}\right] \text { and } \\
\mathbb{E}\left[X_{i} 1_{\{A(\epsilon)\}} \mid X_{n}=u(1-\epsilon)\right] & =\mathbb{E}\left[X_{i}(u(1-(n-1) \epsilon)) 1_{\{A(\epsilon, u(1-(n-1) \epsilon))\}}\right] .
\end{aligned}
$$


We will assume that $\rho_{1, n}>0$, the other cases are analogous. From Proposition 4.2 we get that for $x_{0}=\frac{\rho \sqrt{1-\rho^{2}}}{\alpha+\rho^{2}-1}$ and $\delta>0$,

$$
\begin{aligned}
\mathbb{E}\left[X_{1} \mid X_{n}=u\right] & \sim \mathbb{E}\left[X_{1}(u) 1_{\left\{\left|Y_{1}-\bar{u} x_{0}\right|<\delta \bar{u}\right\}}\right] \\
& \sim\left(1+o_{\epsilon}(1)\right) \mathbb{E}\left[X_{1}(u(1-(n-1) \epsilon)) 1_{\left\{\left|Y_{1}-\bar{u} x_{0}\right|<\delta \bar{u}\right\}}\right] .
\end{aligned}
$$

We want to show that uniformly on $\left\{\left|Y_{1}-\bar{u} x_{0}\right|<\delta \bar{u}\right\}$,

$$
\lim _{u \rightarrow \infty} \frac{X_{1}(u)}{X_{1}(u(1-(n-1) \epsilon))}=1+o_{\epsilon}(1) .
$$

With $y:=\sqrt{1-\rho^{2}} Y_{1} / \bar{u}$ we get

$$
\frac{X(u)}{X(u(1-(n-1) \epsilon))} \sim \frac{F^{-1}\left(1-\frac{(\sqrt{2 \pi} \bar{u} \bar{F}(u))^{(y+\rho)^{2}}}{(\rho+y) \sqrt{2 \pi} \bar{u}}\right)}{F^{-1}\left(1-\frac{\left(\sqrt{2 \pi} \frac{\bar{u}}{u(1-(n-1) \epsilon) \bar{F}(u(1-(n-1) \epsilon)))^{\left(y \frac{\bar{u}}{u(1-(n-1) \epsilon)}+\rho\right)^{2}}}\right.}{\left(\rho+y \frac{\bar{u}}{\overline{u(1-(n-1) \epsilon)}}\right) \sqrt{2 \pi} \overline{u(1-(n-1) \epsilon)}}\right)} .
$$

Define $g(u)$ as in Proposition 4.2. Since $\bar{u}$ is slowly varying and $F^{-1}$ is regularly varying we can concentrate on

$$
\begin{aligned}
& \exp \left(\log (g(u))\left((y+\rho)^{2}-\left(y \frac{\bar{u}}{\overline{u(1-(n-1) \epsilon)}}+\rho\right)^{2}\right)\right. \\
& \left.-\log \left(\frac{g(u(1-(n-1) \epsilon))}{g(u)}\right)\left(y \frac{\bar{u}}{\overline{u(1-(n-1) \epsilon)}}+\rho\right)^{2}\right) .
\end{aligned}
$$

Since $g(u)$ is regularly varying, we have that

$$
\lim _{u \rightarrow \infty} \log \left(\frac{g(u(1-(n-1) \epsilon))}{g(u)}\right)\left(y \frac{\bar{u}}{\overline{u(1-(n-1) \epsilon)}}+\rho\right)^{2}=0 .
$$

Further we have that for $1<\xi_{u, \epsilon}<\frac{\bar{u}}{\overline{u(1-(n-1) \epsilon)}}$,

$$
\begin{aligned}
& \log (g(u))\left((y+\rho)^{2}-\left(y \frac{\bar{u}}{\overline{u(1-(n-1) \epsilon)}}+\rho\right)^{2}\right) \\
& =\log (g(u)) \frac{\bar{u}-\overline{u(1-\epsilon)}}{\overline{u(1-(n-1) \epsilon)}} 2 y\left(y\left(1-\xi_{u, \epsilon}\right)+\rho\right) \\
& \sim 2 \log (g(u)) \frac{\bar{u}-\overline{u(1-(n-1) \epsilon)}}{\overline{u(1-(n-1) \epsilon)}} y(y+\rho) .
\end{aligned}
$$


Now Eq. 19 follows with $0<\hat{\xi}_{u, \epsilon}<(n-1) \epsilon$

$$
\begin{aligned}
& \frac{2 \log (g(u))}{\overline{u(1-(n-1) \epsilon)}}\left(\Phi^{-1}(F(u))-\Phi^{-1}(F(u(1-\epsilon)))\right) \\
& \quad \sim-(n-1) \epsilon \Phi^{-1}\left(F(u(1-\epsilon)) \frac{u f\left(u\left(1-\hat{\xi}_{u, \epsilon}\right)\right.}{\phi\left(\Phi ^ { - 1 } \left(F\left(u\left(1-\hat{\xi}_{u, \epsilon}\right)\right)\right.\right.} \sim \frac{(n-1) \epsilon}{1-\hat{\xi}_{u, \epsilon}} .\right.
\end{aligned}
$$

It follows that

$$
\begin{aligned}
\mathbb{E}[ & \left.X_{i}(u(1-(n-1) \epsilon)) 1_{\{A(\epsilon, u(1-(n-1) \epsilon))\}}\right] \\
\sim & \left(1+o_{\epsilon}(1)\right) \mathbb{E}\left[X_{i}(u) 1_{\{A(\epsilon, u(1-(n-1) \epsilon))\}} 1_{\left\{\left|Y_{1}-\bar{u} x_{0}\right|<\delta \bar{u}\right\}}\right] \\
= & \left(1+o_{\epsilon}(1)\right)\left(\mathbb{E}\left[X_{i}(u) 1_{\{A(\epsilon, u)\}} 1_{\left\{\left|Y_{1}-\bar{u} x_{0}\right|<\delta \bar{u}\right\}}\right]\right. \\
& \left.+\mathbb{E}\left[X_{i}(u) 1_{\left\{\left|Y_{1}-\bar{u} x_{0}\right|<\delta \bar{u}\right\}}\left(1_{\{A(\epsilon, u(1-(n-1) \epsilon))\}}-1_{\{A(\epsilon, u)\}}\right)\right]\right) .
\end{aligned}
$$

Hence it is left to show that

$$
\begin{aligned}
\mathbb{E} & {\left[X_{i}(u) 1_{\left\{\left|Y_{1}-\bar{u} x_{0}\right|<\delta \bar{u}\right\}}\left|1_{\{A(\epsilon, u(1-(n-1) \epsilon))\}}-1_{\{A(\epsilon, u)\}}\right|\right] } \\
= & : \mathbb{E}\left[X_{i}(u) 1_{\left\{\left|Y_{1}-\bar{u} x_{0}\right|<\delta \bar{u}\right\}} 1_{\{A(\epsilon, u(1-(n-1) \epsilon)) \triangle A(\epsilon, u)\}}\right]=o\left(\mathbb{E}\left[X_{1} \mid X_{n}=u\right]\right) .
\end{aligned}
$$

Define

$$
\begin{aligned}
B(\epsilon, u):= & \left\{\sum_{i=1}^{n-1} \sigma_{i, n}^{-1} \Phi^{-1}\left(F\left(X_{i}(u)\right)\right)<0\right\} \\
& \triangle\left\{\sum_{i=1}^{n-1} \sigma_{i, n}^{-1} \Phi^{-1}\left(F\left(X_{i}(u(1-(n-1) \epsilon))\right)\right)<0\right\} \\
B_{i}(\epsilon, u):= & \left\{X_{i}(u) \leq \epsilon\right\} \triangle\left\{X_{i}(u(1-(n-1) \epsilon)) \leq \epsilon\right\}, \quad i=1, \ldots, n-1 .
\end{aligned}
$$

Note that

$$
A(\epsilon, u(1-(n-1) \epsilon)) \triangle A(\epsilon, u) \subseteq B(u, \epsilon) \cup \bigcup_{i=1}^{n-1} B_{i}(u, \epsilon)
$$

$B(u, \epsilon)$ can be written as

$$
\begin{aligned}
Y_{n-1} \in & \{x: \overline{u(1-(n-1) \epsilon)}<x<\bar{u}\} \\
& \cdot \frac{1}{-\hat{\rho}_{n-1, n-1}}\left(\sum_{i=1}^{n-1} \sigma_{i, n}^{-1} \rho_{1, n}\right)-\sum_{i=1}^{n-2} \sigma_{i, n}^{-1} \sum_{j=1}^{i} \frac{\hat{\rho}_{i, j}}{\hat{\rho}_{n-1, n-1}} Y_{j}=: \hat{B} .
\end{aligned}
$$


It follows that

$$
\begin{aligned}
\mathbb{E} & {\left[X_{i}(u) 1_{\left\{\left|Y_{1}-\bar{u} x_{0}\right|<\delta \bar{u}\right\}} 1_{\{B(u, \epsilon\}}\right] } \\
][3 p t] & =\int_{\bar{u}\left(x_{0}-\delta\right)}^{\bar{u}\left(x_{0}+\delta\right)} \int_{-\infty}^{\infty} \cdots \int_{-\infty}^{\infty} \int_{y_{n-1} \in \hat{B}}(2 \pi)^{-(n-1) / 2} X_{1}(u) e^{-\sum_{i=1}^{n-1} \frac{x^{2}}{2}} \mathrm{~d} y_{1} \cdots \mathrm{d} y_{n-1} \\
& \leq|\hat{B}| \int_{\bar{u}\left(x_{0}-\delta\right)}^{\bar{u}\left(x_{0}+\delta\right)} \int_{-\infty}^{\infty} \cdots \int_{-\infty}^{\infty}(2 \pi)^{-(n-2) / 2} X_{1}(u) e^{-\sum_{i=1}^{n-2} \frac{x^{2}}{2}} \mathrm{~d} y_{1} \cdots \mathrm{d} y_{n-2} \\
& =|\hat{B}| \mathbb{E}\left[X_{i}(u) 1_{\left\{\left|Y_{1}-\bar{u} x_{0}\right|<\delta \bar{u}\right\}}\right]=o\left(\mathbb{E}\left[X_{i}(u) 1_{\left\{\left|Y_{1}-\bar{u} x_{0}\right|<\delta \bar{u}\right\}}\right]\right),
\end{aligned}
$$

where the last equality follows from

$$
|\hat{B}|=\left|\frac{1}{-\hat{\rho}_{n-1, n-1}}\left(\sum_{i=1}^{n-1} \sigma_{i, n}^{-1} \rho_{1, n}\right)\right|(\bar{u}-\overline{u(1-(n-1) \epsilon)}) \rightarrow 0, \quad \text { as } \quad u \rightarrow \infty .
$$

Since $B_{i}(u, \epsilon)$ can be written as

$$
Y_{i} \in\{x: \overline{u(1-(n-1) \epsilon)}<x<\bar{u}\} \frac{\rho_{i, n}}{-\hat{\rho}_{i, i}}-\frac{1}{\hat{\rho}_{i, i}} \Phi^{-1}(F(\epsilon))-\sum_{j=1}^{i} \frac{\hat{\rho}_{i, j}}{\hat{\rho}_{i, j}} Y_{j},
$$

we can show analogously for $i>2$ that

$$
\mathbb{E}\left[X_{i}(u) 1_{\left\{\left|Y_{1}-\bar{u} x_{0}\right|<\delta \bar{u}\right\}} 1_{\left\{B_{i}(u, \epsilon\}\right.}\right]=o\left(\mathbb{E}\left[X_{i}(u) 1_{\left\{\left|Y_{1}-\bar{u} x_{0}\right|<\delta \bar{u}\right\}}\right]\right) .
$$

For $i=1$ we just have to note that for $Y_{1} \in B_{i}(u, \epsilon)$ it follows that $Y_{1} \sim-\frac{\rho}{\sqrt{1-\rho^{2}}} \bar{u}$ and hence

$$
\lim _{u \rightarrow \infty} 1_{\left\{\left|Y_{1}-\bar{u} x_{0}\right|<\delta \bar{u}\right\}} 1_{\left\{B_{1}(u, \epsilon\}\right.}=0 .
$$

Hence Proposition 4.1 follows.

\section{Appendix C: Proof of Proposition 5.1}

Proof of Proposition 5.1 For the proof we assume that $c=1$ since $c \varphi(x)$ is the generator of the same copula. Note that it follows from the conditions that

- $\varphi(x)=(1-x)+(1-x)^{\beta} L_{1}(1-x)$, where $L_{1}(1 / x)$ is slowly varying and

$$
L(\varphi(x))=-\frac{(1-x)^{\beta}}{\varphi(x)^{\beta}} L_{1}(1-x)
$$

- $\varphi^{\prime}(x)=-1-\beta(1-x)^{\beta-1} L_{3}(1-x)$, where $\lim _{x \rightarrow 0} L_{1}(x) / L_{3}(x)=1$. 
Now $C$ has the continuous density

$$
C_{1 \cdots n}\left(x_{1}, \ldots, x_{n}\right)=\left(\varphi^{-1}\right)^{(n)}\left(\sum_{i=1}^{n} \varphi\left(x_{i}\right)\right) \prod_{i=1}^{n} \varphi^{\prime}\left(x_{i}\right) .
$$

For Assumption 2.3 note:

$$
\begin{aligned}
\mathbb{P}\left(X_{1}>u, X_{2}>u\right) & =1-2 F(u)+C(F(u), F(u)) \\
& =2 \bar{F}(u)-\left(1-\varphi^{-1}(2 \varphi(F(u)))\right) \\
& =2 \bar{F}(u)-2 \varphi(F(u))-(2 \varphi(F(u)))^{\beta} L(2 \varphi(F(u))) \\
& =-2 \bar{F}(u)^{\beta} L_{1}(\bar{F}(u))-2^{\beta}(\varphi(F(u)))^{\beta} L(2 \varphi(F(u))) \\
& \sim\left(2^{\beta}-2\right) \bar{F}(u)^{\beta} L_{1}(\bar{F}(u)) .
\end{aligned}
$$

Hence $\mathbb{P}\left(X_{1}>u, X_{2}>u\right)$ is regularly varying with index $-\alpha \beta$, which leads to $\rho=\frac{2}{\beta}-1$. Assumption 2.1: Chose $0<\epsilon_{1}<1$ and $0<\epsilon_{2}$ then uniformly for $\epsilon_{1}<y<\left(1+\epsilon_{2}\right)$ and $a \rightarrow 0$

$$
\begin{aligned}
& \frac{\int_{1-a}^{1} C_{1,2}(x, 1-y a) \mathrm{d} x}{\int_{1-a}^{1} C_{1,2}(x, 1-a) \mathrm{d} x} \\
& =\frac{\left(\varphi^{-1}\right)^{\prime}(\varphi(1-y a))-\left(\varphi^{-1}\right)^{\prime}(\varphi(1-a)+\varphi(1-y a))}{\left(\varphi^{-1}\right)^{\prime}(\varphi(1-a))-\left(\varphi^{-1}\right)^{\prime}(\varphi(1-a)+\varphi(1-a))} \\
& =\frac{\beta(\varphi(1-a)+\varphi(1-y a))^{\beta-1} L_{2}(\varphi(1-a)+\varphi(1-y a))-\beta(\varphi(1-y a))^{\beta-1} L_{2}(\varphi(1-y a))}{\beta(\varphi(1-a)+\varphi(1-a))^{\delta-1} L_{2}(\varphi(1-a)+\varphi(1-a))-\beta(\varphi(1-a))^{\beta-1} L_{2}(\varphi(1-a))} \\
& =\frac{\left(\frac{\varphi(1-y a)}{\varphi(1-a)}+1\right)^{\beta-1} L_{2}(\varphi(1-y a)+\varphi(1-a))-\frac{\varphi(1-y a)}{\varphi(1-a)} L_{2}(\varphi(1-y a))}{2^{\beta-1} L_{2}(2 \varphi(1-a))-L_{2}(\varphi(1-a))} \\
& \sim \frac{(y+1)^{\beta-1}-y}{2^{\beta-1}-1},
\end{aligned}
$$

hence Assumption 2.1 holds.

For Assumptions 2.2 note that:

$$
\begin{aligned}
& \frac{\int_{1-y a}^{1} C_{1,2}(x, 1-a) \mathrm{d} x}{\int_{1-\delta a}^{1} C_{1,2}(x, 1-a) \mathrm{d} x} \\
& =\frac{\left(\varphi^{-1}\right)^{\prime}(\varphi(1-a))-\left(\varphi^{-1}\right)^{\prime}(\varphi(1-y a)+\varphi(1-a))}{\left(\varphi^{-1}\right)^{\prime}(\varphi(1-a))-\left(\varphi^{-1}\right)^{\prime}(\varphi(1-\delta a)+\varphi(1-a))} \\
& =\frac{\left(\frac{\varphi(1-y a)}{\varphi(1-a)}+1\right)^{\beta-1} L_{2}(\varphi(1-y a)+\varphi(1-a))-L_{2}(\varphi(1-a))}{\left(\frac{\varphi(1-\delta a)}{\varphi(1-a)}+1\right)^{\beta-1} L_{2}(\varphi(1-\delta a)+\varphi(1-a))-L_{2}(\varphi(1-a))} .
\end{aligned}
$$


Next, for any $\delta<M$ we get uniformly in $\delta<y<M$ that

$$
\begin{aligned}
& \lim _{u \rightarrow 0} \frac{\left(\frac{\varphi(1-y a)}{\varphi(1-a)}+1\right)^{\beta-1} L_{2}(\varphi(1-y a)+\varphi(1-a))-L_{2}(\varphi(1-a))}{\left(\frac{\varphi(1-\delta a)}{\varphi(1-a)}+1\right)^{\beta-1} L_{2}(\varphi(1-\delta a)+\varphi(1-a))-L_{2}(\varphi(1-a))} \\
& =\frac{(y+1)^{\beta-1}-1}{(\delta+1)^{\beta-1}-1} .
\end{aligned}
$$

Hence we can choose $\gamma_{1}=\gamma_{2}=\beta-1>1 / \alpha$.

By Taylor theorem it follows that for $0<\xi_{y}<\varphi(1-y a)$

$$
\begin{aligned}
& \frac{\left(\varphi^{-1}\right)^{\prime}(\varphi(1-a))-\left(\varphi^{-1}\right)^{\prime}(\varphi(1-y a)+\varphi(1-a))}{\left(\varphi^{-1}\right)^{\prime}(\varphi(1-a))-\left(\varphi^{-1}\right)^{\prime}(\varphi(1-\delta a)+\varphi(1-a))} \\
& =\frac{\varphi(1-y a)}{\varphi(1-\delta a)} \frac{\left(\varphi^{-1}\right)^{\prime \prime}\left(\varphi(1-a)+\xi_{y}\right)}{\left(\varphi^{-1}\right)^{\prime \prime}\left(\varphi(1-a)+\xi_{0}\right)} .
\end{aligned}
$$

Since $\varphi$ and $\left(\varphi^{-1}\right)^{\prime \prime}$ are regularly varying we can choose $\gamma_{3}=1$ and Assumption 2.2 follows.

To prove Assumption 3.2 at first note that uniformly for $0<x<1 / 2$

$$
\lim _{u \rightarrow \infty} \frac{\varphi^{\prime}(F(u(1-x))}{\varphi^{\prime}(F(u))}=1
$$

and

$$
\begin{aligned}
& \frac{C_{1 \cdots n}\left(F\left(u y_{1}\right), \ldots, F\left(u y_{n-1}\right), F(u(1-x))\right)}{C_{1 \cdots n}\left(F\left(u y_{1}\right), \ldots, F\left(u y_{n-1}\right), F(u)\right)} \\
& =\frac{\left(\varphi^{-1}\right)^{(n)}\left(\sum_{i=1}^{n-1} \varphi\left(F\left(u y_{i}\right)\right)+\varphi(F(u(1-x)))\right) \varphi^{\prime}(F(u(1-x)))}{\left(\varphi^{-1}\right)^{(n)}\left(\sum_{i=1}^{n-1} \varphi\left(F\left(u y_{i}\right)\right)+\varphi(F(u))\right) \varphi^{\prime}(F(u))} .
\end{aligned}
$$

Since $\left|\left(\varphi^{-1}\right)^{(n)}\right|$ and $\varphi$ are monotone decreasing and $F$ is monotone increasing, we get that

$$
\begin{aligned}
& \left|\left(\varphi^{-1}\right)^{(n)}\left(\sum_{i=1}^{n-1} \varphi\left(F\left(u y_{i}\right)\right)+\varphi(F(u(1-\epsilon)))\right)\right| \\
& \leq\left|\left(\varphi^{-1}\right)^{(n)}\left(\sum_{i=1}^{n-1} \varphi\left(F\left(u y_{i}\right)\right)+\varphi(F(u(1-x)))\right)\right| \\
& \leq\left|\left(\varphi^{-1}\right)^{(n)}\left(\sum_{i=1}^{n-1} \varphi\left(F\left(u y_{i}\right)\right)+\varphi(F(u))\right)\right| .
\end{aligned}
$$


It follows that for $A(\epsilon)=\emptyset$ Assumption 3.2 is fulfilled. Finally for Assumption 3.1 note that

$$
\begin{aligned}
\mathbb{E}\left[X_{1} \mid X_{2}=u\right] & =\varphi^{\prime}(F(u)) \int_{0}^{\infty} x\left(\varphi^{-1}\right)^{\prime \prime}(\varphi(F(x))+\varphi(F(u))) \varphi^{\prime}(F(x)) f(x) \mathrm{d} x \\
& \sim-\frac{1}{c} \int_{0}^{\infty} x\left(\varphi^{-1}\right)^{\prime \prime}(\varphi(F(x))+\varphi(F(u))) \varphi^{\prime}(F(x)) f(x) \mathrm{d} x \\
& \leq-\frac{1}{c} \int_{0}^{\infty} x\left(\varphi^{-1}\right)^{\prime \prime}(\varphi(F(x))) \varphi^{\prime}(F(x)) f(x) \mathrm{d} x .
\end{aligned}
$$

Since

$$
\left(\varphi^{-1}\right)^{\prime \prime}(\varphi(F(x))) \varphi^{\prime}(F(x)) \approx-\frac{1}{c} \bar{F}(x)^{\beta-2},
$$

we get that

$$
\lim _{u \rightarrow \infty} \mathbb{E}\left[X_{1} \mid X_{2}=u\right]=-\frac{1}{c} \int_{0}^{\infty} x\left(\varphi^{-1}\right)^{\prime \prime}(\varphi(F(x)))\left(\varphi^{\prime}(F(x))\right) f(x) \mathrm{d} x<\infty .
$$

\section{Appendix D: Proof of Proposition 6.1}

Proof of Proposition 6.1 Since $C_{1 \ldots n}$ is bounded and hence uniformly continuous we get that

$$
\mathbb{P}\left(X_{i}>u, X_{j}>u\right)=\int_{F(u)}^{1} \int_{F(u)}^{1} C_{i, j}^{m}\left(x_{i}, x_{j}\right) \mathrm{d} x_{i} \mathrm{~d} x_{j} \sim \bar{F}(u)^{2} C_{i j}^{m}(1,1)
$$

hence Assumptions 2.3 holds with $\rho_{i, j}=0$. Assumptions 2.1 follows from

$$
\int_{1-a}^{1} C_{i j}^{m}(x, 1-y a) \mathrm{d} x \leq M \int_{1-a}^{1} \mathrm{~d} x \leq \frac{M}{m} \int_{1-a}^{1} C_{i j}^{m}(x, 1-a) \mathrm{d} x
$$

Assumptions 2.2 follow from

$$
\frac{m}{M} \frac{y}{\delta}=\frac{\int_{1-y a}^{1} m \mathrm{~d} x}{\int_{1-\delta a}^{1} M \mathrm{~d} x} \leq \frac{\int_{1-y a}^{1} C_{i j}^{m}(x, 1-a) \mathrm{d} x}{\int_{1-\delta a}^{1} C_{i j}^{m}(x, 1-a) \mathrm{d} x} \leq \frac{\int_{1-y a}^{1} M \mathrm{~d} x}{\int_{1-\delta a}^{1} m \mathrm{~d} x}=\frac{M}{m} \frac{y}{\delta}
$$

Since $C_{1 \cdots n}$ is uniformly continuous we get that Assumption 3.2 is fulfilled with $A(\epsilon):=\emptyset$ and Assumption 3.1 follows from

$$
\lim _{a \rightarrow 0} \frac{\int_{0}^{1} F^{-1}(x) C_{i j}^{m}(x, 1-(1+\epsilon) a) \mathrm{d} x}{\int_{0}^{1} F^{-1}(x) C_{i j}^{m}(x, 1-a) \mathrm{d} x}=\frac{\int_{0}^{1} F^{-1}(x) C_{i j}^{m}(x, 1) \mathrm{d} x}{\int_{0}^{1} F^{-1}(x) C_{i j}^{m}(x, 1) \mathrm{d} x}=1 .
$$




\section{References}

Albrecher, H., Asmussen, S., Kortschak, D.: Tail asymptotics for the sum of two heavy-tailed dependent risks. Extremes 9(2), 107-130 (2006)

Albrecher, H., Hipp, C., Kortschak, D.: Higher-order expansions for compound distributions and ruin probabilities with subexponential claims. Scand. Actuar. J. 2010(2), 105-135 (2010)

Asmussen, S., Kroese, D.P.: Improved algorithms for rare event simulation with heavy tails. Adv. Appl. Probab. 38(2), 545-558 (2006)

Barbe, P., McCormick, W.P.: Asymptotic Expansions for Infinite Weighted Convolutions of Heavy Tail Distributions and Applications. American Mathematical Society, Providence (2009)

Bingham, N.H., Goldie, C.M., Teugels, J.L.: Regular variation. In: Encyclopedia of Mathematics and its Applications, vol. 27. Cambridge University Press, Cambridge (1989)

Coles, S., Heffernan, J., Tawn, J.: Dependence measures for extreme value analyses. Extremes 2(4), 339365 (1999)

Davis, R.A., Resnick, S.I.: Limit theory for bilinear processes with heavy-tailed noise. Ann. Appl. Probab. 6(4), 1191-1210 (1996)

de Haan, L., de Ronde, J.: Sea and wind: multivariate extremes at work. Extremes 1(1), 7-45 (1998)

de Haan, L., Resnick, S.I.: Estimating the limit distribution of multivariate extremes. Commun. Stat., Stoch. Models 9(2), 275-309 (1993)

Geluk, J.L.: Second order tail behaviour of a subordinated probability distribution. Stoch. Process. Their Appl. 40(2), 325-337 (1992)

Geluk, J., de Haan, L., Resnick, S., Stărică, C.: Second-order regular variation, convolution and the central limit theorem. Stoch. Process. Their Appl. 69(2), 139-159 (1997)

Ledford, A.W., Tawn, J.A.: Statistics for near independence in multivariate extreme values. Biometrika 83(1), 169-187 (1996)

Mitra, A., Resnick, S.I.: Aggregation of rapidly varying risks and asymptotic independence. Adv. Appl. Probab. 41(3), 797-828 (2009)

Nelsen, R.B.: An Introduction to Copulas. Springer Series in Statistics, 2nd edn. Springer, New York (2006)

Omey, E., Willekens, E.: Second order behaviour of the tail of a subordinated probability distribution. Stoch. Process. Their Appl. 21(2), 339-353 (1986)

Resnick, S.: Hidden regular variation, second order regular variation and asymptotic independence. Extremes 5(4), 303-336 (2002) 